Delft University of Technology

\title{
Wing Stiffness and Hinge Release Threshold Effects on Folding Wingtip Gust Load Alleviation
}

Carrillo Córcoles, X.; Mertens, C.; Sciacchitano, A.; van Oudheusden, B.W.; De Breuker, R.; Sodja, J.

DOI

10.2514/6.2022-1559

Publication date

2022

Document Version

Final published version

Published in

AIAA SCITECH 2022 Forum

\section{Citation (APA)}

Carrillo Córcoles, X., Mertens, C., Sciacchitano, A., van Oudheusden, B. W., De Breuker, R., \& Sodja, J. (2022). Wing Stiffness and Hinge Release Threshold Effects on Folding Wingtip Gust Load Alleviation. In AIAA SCITECH 2022 Forum [AIAA 2022-1559] (AIAA Science and Technology Forum and Exposition, AIAA SciTech Forum 2022). https://doi.org/10.2514/6.2022-1559

Important note

To cite this publication, please use the final published version (if applicable).

Please check the document version above.

\footnotetext{
Copyright

Other than for strictly personal use, it is not permitted to download, forward or distribute the text or part of it, without the consent of the author(s) and/or copyright holder(s), unless the work is under an open content license such as Creative Commons.

Takedown policy

Please contact us and provide details if you believe this document breaches copyrights.

We will remove access to the work immediately and investigate your claim.
} 


\title{
Wing Stiffness and Hinge Release Threshold Effects on Folding Wingtip Gust Load Alleviation
}

\author{
Xavier Carrillo Córcoles*, Christoph Mertens ${ }^{\dagger}$, Andrea Sciacchitano ${ }^{\ddagger}$, Bas W. van Oudheusden ${ }^{\S}$, Roeland De \\ Breuker "II and Jurij Sodjall
}

An aeroelastic wind tunnel experiment to identify the influence of the wing stiffness and hinge release threshold on the gust load alleviation performance of a folding wingtip design is presented in this study. Five models with different stiffness and tailoring properties are tested and the wing root bending moment at different conditions is compared to the response with locked hinge conditions to assess the impact on the gust load alleviation capabilities of the folding wingtip. The results show that the structural properties do not have an important impact on the peak load alleviation but the hinge release threshold and timing do. Releasing with the correct timing can reduce significantly the peak loads. However, the dynamics of the system are affected by this release: the flutter speed is decreased and, although the performance can improve, load oscillations increase, which can be considered detrimental for reasons such as fatigue.

Variables

\section{Nomenclature}

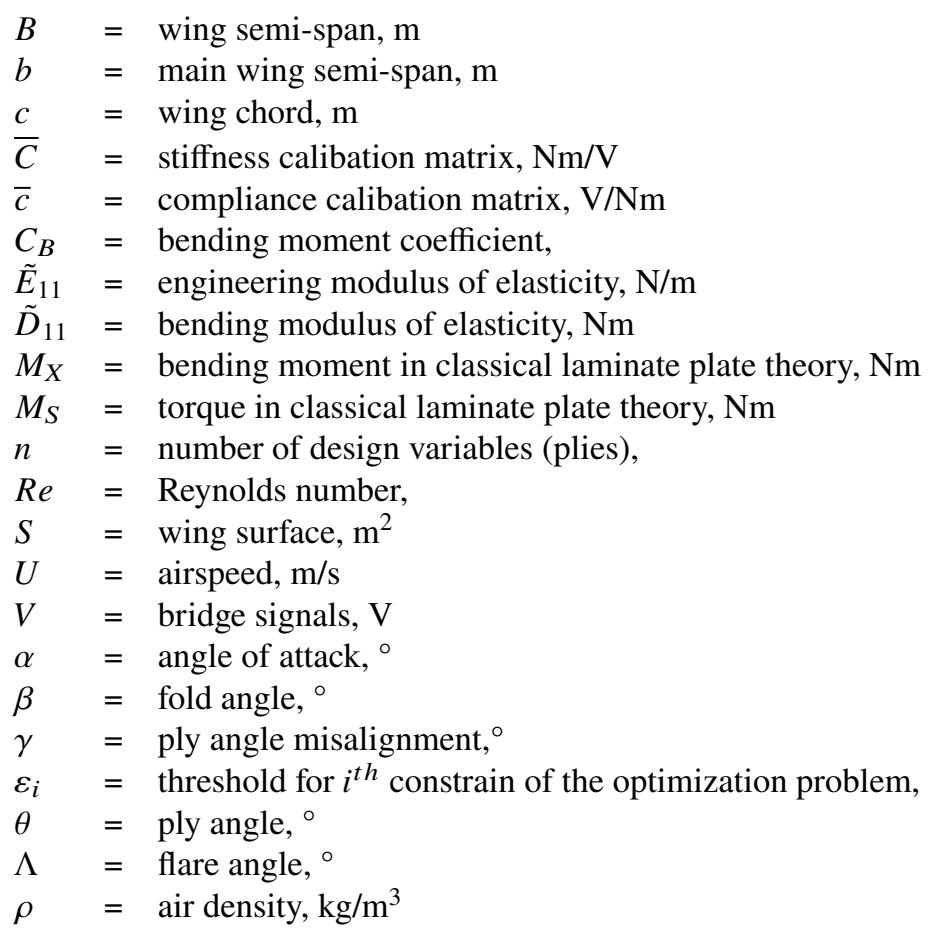

\footnotetext{
*MSc Student, Faculty of Aerospace Engineering, Aerodynamics, Kluyverweg 22629 HS Delft, X.CarrilloCorcoles@student.tudelft.nl

$\dagger \mathrm{PhD}$ Candidate, Faculty of Aerospace Engineering, Aerodynamics, Kluyverweg 22629 HS Delft, C.Mertens@ tudelft.nl

$¥$ Assistant Professor, Faculty of Aerospace Engineering, Aerodynamics, Kluyverweg 22629 HS Delft, A.Sciacchitano@tudelft.nl

$\S$ Associate Professor, Faculty of Aerospace Engineering, Aerodynamics, Kluyverweg 22629 HS Delft, B.W.vanOudheusden@ tudelft.nl

II Associate Professor, Faculty of Aerospace Engineering, Aerospace Structures and Computational Mechanics, Kluyverweg 12629 HS Delft, R.deBreuker@tudelft.nl, AIAA Associate Fellow

"Senior Scientist, Faculty of Aerospace Engineering, Aerospace Structures and Computational Mechanics, Kluyverweg 12629 HS Delft, J.Sodja@tudelft.nl, AIAA Member
} 


\section{List of abbreviations}

$\begin{array}{ll}\text { AR } & =\text { Aspect Ratio } \\ \text { DLM } & =\text { Doublet Lattice Method } \\ \text { FEM } & =\text { Finite Element Model } \\ \text { FRF } & =\text { Frequency Response Function } \\ \text { FWT } & =\text { Folding Wingtip } \\ \text { GA } & =\text { Genetic Algorithm } \\ \text { GLA } & =\text { Gust Load Alleviation } \\ \text { LCO } & =\text { Limit Cycle Oscillation } \\ \text { LS } & =\text { Least-Squares } \\ \text { RMS } & =\text { Root Mean Square } \\ \text { WRBM } & =\text { Wing Root Bending Moment }\end{array}$

\section{Introduction}

IRCRAFt aerodynamic efficiency can be improved by increasing the wingspan and thereby the aspect ratio of the main wing. However, increasing the wingspan can lead to increased structural weight of the wing due to increased structural loads in the inboard section of the wing, reduced handling qualities due to reduced roll rate, and operational difficulties due to limited parking space at the airports. [1]]

To address the operational difficulties, Boeing [2] proposed a folding wingtip (FWT) which can be folded when the airplane is on the ground and deployed during flight. On the other hand, Wilson et al. [3] proposed a FWT using a flared hinge, which can be released in flight if necessary, to address also the structural and handling drawbacks due to the span increase of the wing.

The numerical analyses presented by Castrichini et al. [4] showed that the FWT can reduce the wing root bending moment (WRBM) and provide a means of gust load alleviation (GLA). These results were confirmed in wind tunnel tests. Cheung et al. [5, 6] showed the potential for passive load alleviation of the system, achieving peak load reductions between $30 \%$ and $60 \%$, and studied the active control of the FWT, which, depending on the timing of the actuation with respect to the gusts, can increase the GLA performance up to $80 \%$ reduction. This timing issue is also important when releasing the hinge, since the release instant affects the magnitude of the load alleviation, as reported by Castrichini et al. [7], 8].

Regarding the handling qualities, first Dussart et al. [9] in numerical analyses and later Healy et al. [10] in wind tunnel tests, found that it is possible to decrease the reduction of steady roll rates caused by the increment of the wingspan when the FWT is released. It is possible to achieve between $60 \%$ and $80 \%$ of the roll rate of a wing without extension.

To validate these findings in flight, Wilson et al. [II] used a scaled aircraft model based on the A321, the AlbatrossONE, that includes a hinge mechanism to lock and release the FWT. The results confirmed the potential of the concept for load alleviation, with load reductions between $20 \%$ and $40 \%$ depending on the FWT span, and presented the proof-of-concept with a gate-to-gate demonstration.

In continuation to their work, the current study proposes an aeroelastic experimental investigation to characterize how the load alleviation capabilities of the FWT are affected by the variation of the wing stiffness, passive load alleviation using composite tailoring, and the variation of load threshold for releasing the hinge. Thanks to the introduction of composites, it is possible to manufacture lighter yet more flexible wings and introduce the concept of aeroelastic tailoring, which can be used to optimize the structural response of the wing during maneuvers and gust encounters. Therefore, the stiffness and bend-twist coupling properties of the main wing are varied to investigate their effect on the FWT alleviation performance.

Finally, given the importance of the hinge release timing shown by Castrichini et al. [7, 8], the third parameter that is varied is the load threshold to release the FWT. This variation allows, on the one hand, studying experimentally what was observed in the numerical investigations and, on the other hand, in combination with the previous parameters, studying how the optimal threshold is affected by the structural properties of the main wing. 


\section{Wing Design Study}

In this section, the procedure and methodologies used to design the wind tunnel model are addressed. First, the characteristics of the wing are presented. Then, the finite element model used for sizing the experiment is explained. Next, the design and selection of the wing structure are defined. Finally, the simulations carried out for the sizing are presented.

\section{A. Wind Tunnel Wing Model}

The objective of this study is to establish the relationship between the stiffness properties of the main wing, the load threshold for the hinge release, and the GLA performance of the FWT. For this reason, the model must fulfill two main requirements: (i) a controlled variation of the structural properties and (ii) a mechanism to release the hinge based on the load threshold. In addition to the main functional requirements pertaining to the FWT, the model must allow the observation of aeroelastic effects pertinent to highly flexible wings (e.g. large deflections) in the wind tunnel. However, the large deflections are constrained by the wind tunnel, which constrains the size of the model and the magnitude of the structural deformations.

The model is designed as a rectangular half-wing with a chord, $c$, of $100 \mathrm{~mm}$ and a wing aspect ratio (AR) of 14 to maintain similar proportions as the AlbatrossONE [II] but simplifying the geometry to facilitate the parametric study. The main wing has a semi-span, $b$, of $500 \mathrm{~mm}$ and the wingtip extends the full semi-span, $B$, to a total of 700 $\mathrm{mm}$. In view of the restrictions set by the wing dimensions, a NACA 0018 airfoil is selected, to be able to fit the hinge mechanism inside the contour of the airfoil profile. Finally, the flare angle of the hinge, $\Lambda$, is set to $15^{\circ}$, as in the AlbatrossONE, and the wingtip fold angle is defined as $\beta$. The model and an overview of its dimensions are presented in Figure 1 and Table-1.

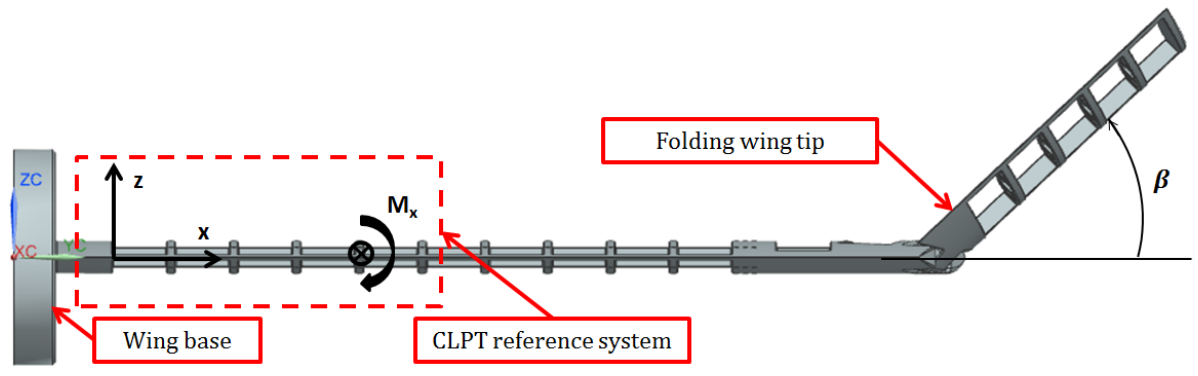

(a) Rear view

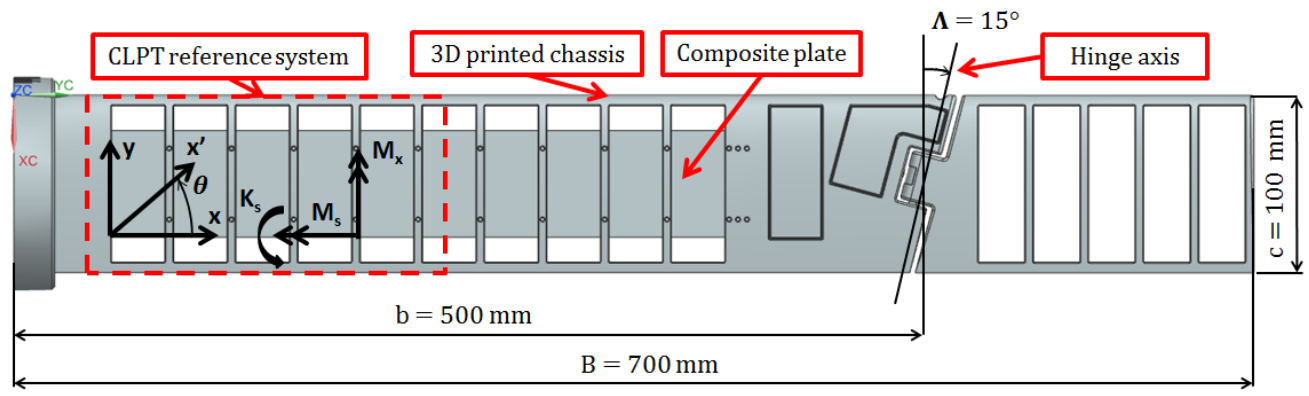

(b) Top View

Fig. 1 CAD representation of the wind tunnel model without skin with an overview of dimensions

With the objective of achieving large deflections at the given size of the model and to easily vary the stiffness properties of the wing, the model is based on the Pazy wing concept proposed by Avin et al. [12]. It consists of a nylon PA12 3D printed chassis ${ }^{\text {Ta }}$ covered with Oralight foil ${ }^{\mathbb{E}}$ to provide the aerodynamic shape to the wing while a plate inside it acts as the primary structural element carrying the majority of the loads. Since the plate represents the primary load-

\footnotetext{
*"PA2200 Product Information", EOS Gmbh, 2001. URL https://cdn2.hubspot.net/hubts/5154612/Material\%20documentation/ Nylon\%20PA12/PA2200_Product_information_03-10_en.pdf, retrieved 28 July 2021

†ralight, Lanitz-Prena Folien Factory Gmbh, 2021. URL https://wWw.oracover.de/katalog/artikelinto/4209/oralightlightiron-on-film---width_-60-cm---1ength_-2-m, retrieved 28 July 2021.
} 
Table 1 Overview of model dimensions

\begin{tabular}{ccc}
\hline \hline Symbol & Description & Dimension \\
\hline$\Lambda$ & Flare angle & $15^{\circ}$ \\
$B$ & Full wing semi-span & $700 \mathrm{~mm}$ \\
$\mathrm{~b}$ & Main wing semi-span & $500 \mathrm{~mm}$ \\
$\mathrm{c}$ & Chord & $100 \mathrm{~mm}$ \\
- & Airfoil & NACA 0018 \\
\hline \hline
\end{tabular}

bearing element of the wing, it is possible to effectively control the structural properties of the wing by only modifying the plate, whose bending stiffness and bend-twist coupling properties can be modified using composite tailoring, which is done with NTPT TP135 prepregt. The sizing of these plates is carried out using numerical simulations, explained in subsection II.C.

Finally, the hinge has to be released at different instants of the gust response (i.e. load thresholds). Therefore, it is necessary to design a mechanism that can easily modify the release time, achieve a reaction time far below the gust period, and operate consistently over the experiment. These requirements can be fulfilled by an active servo-actuated mechanism, which simplifies the assembly and gives flexibility to the release possibilities.

The hinge release mechanism is designed to block the wingtip rotation by creating an interference between the main wing and the FWT. The realization of the mechanism is shown in Figure 2. The locking pin is pressed against the hinge axle of the wingtip using an eccentric lever connected to a Blue Bird BMS-A10H rotary actuator $\$$, which is controlled by an Arduino microcontroller ${ }^{\mathbb{1}}$ that releases the hinge at the desired instant. By adding a spring and a chamfer at the end of the pin, the system is designed in such a way that the FWT can start moving as soon as the pressure on the locking pin is released. This way, even if the pin has not moved to the end of its stroke, the FWT can already rotate freely. In addition, as a result of the axial alignment of the reaction force of the pin and the eccentric lever, the actuator does not have to actively resist the reaction load on the locking pin.

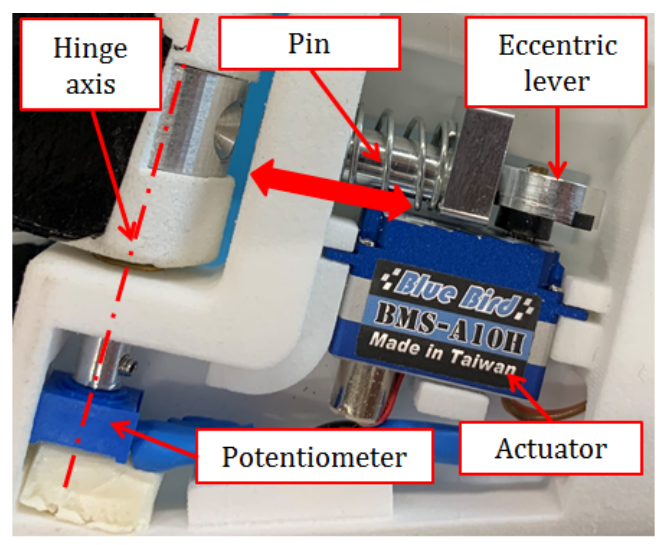

Fig. 2 Detail of the hinge mechanism in free-hinge condition

\section{B. Finite Element Model}

The structural design of the wings, in particular the layup of the composite plates acting as the main load-bearing components, requires an assessment of the aeroelastic response to ensure that the desired aeroelastic phenomena can be observed in the wind tunnel. Therefore, the anticipated loads and deflections during the wind tunnel test are studied

†P135 Data Sheet, North Thin Ply Technology, 2019. URL https://www.thınplytechnology.com/assets/mesimages/NTPTTDSTP135_V1-6.pdI, retrieved 28 July 2021.

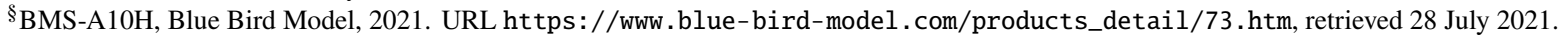

"IIArduino Boards and Modules, Arduino, 2021. URL https://store.arduino.cc/collections/boards, retrieved 25 May 2021. 


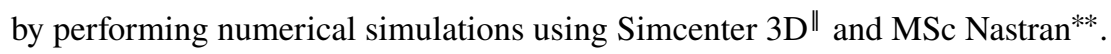

The structure of the wing is modeled using the approach presented by Avin et al. [12]. The leading and trailing edges are modeled as 1D beams while the ribs and the plate are modeled as 2D shells. For the ribs at end of the main wing and the wingtip, where there is no inner plate, ribs are modeled as a single surface. For the other ribs, only the front $\mathrm{D}$ and rear triangle are modeled and connected to each other with rectangular caps modeled as $1 \mathrm{D}$ beams. The glue bonding between the ribs and the plate is modeled using RBE2 1D elements. Finally, the wing skin is modeled as 2D shells. Figure 3 shows the FEM representation of the wing without skin.

As a simplification of the problem, Avin et al. [12] only modeled the skin when subjected to tensile loads, since buckling of the Oralight skin under compressive loads introduces nonlinearities to the analysis. However, both skins are modeled in this sizing study, as the oscillatory motion in the gust response makes both skins face compressive and tensional loads. The simulations are carried out with and without the skin to determine the upper and the lower envelope of the expected loads and deflections. The results without skin are considered conservative since, in the real structure, the skin will add to the stiffness of the structure and thereby also carry some of the loads. Similarly, the results including both the upper and the bottom skins are non-conservative, as the model becomes stiffer than the real structure when the nonlinearities are ignored. Hence, these results set a range in which the real response can be expected.

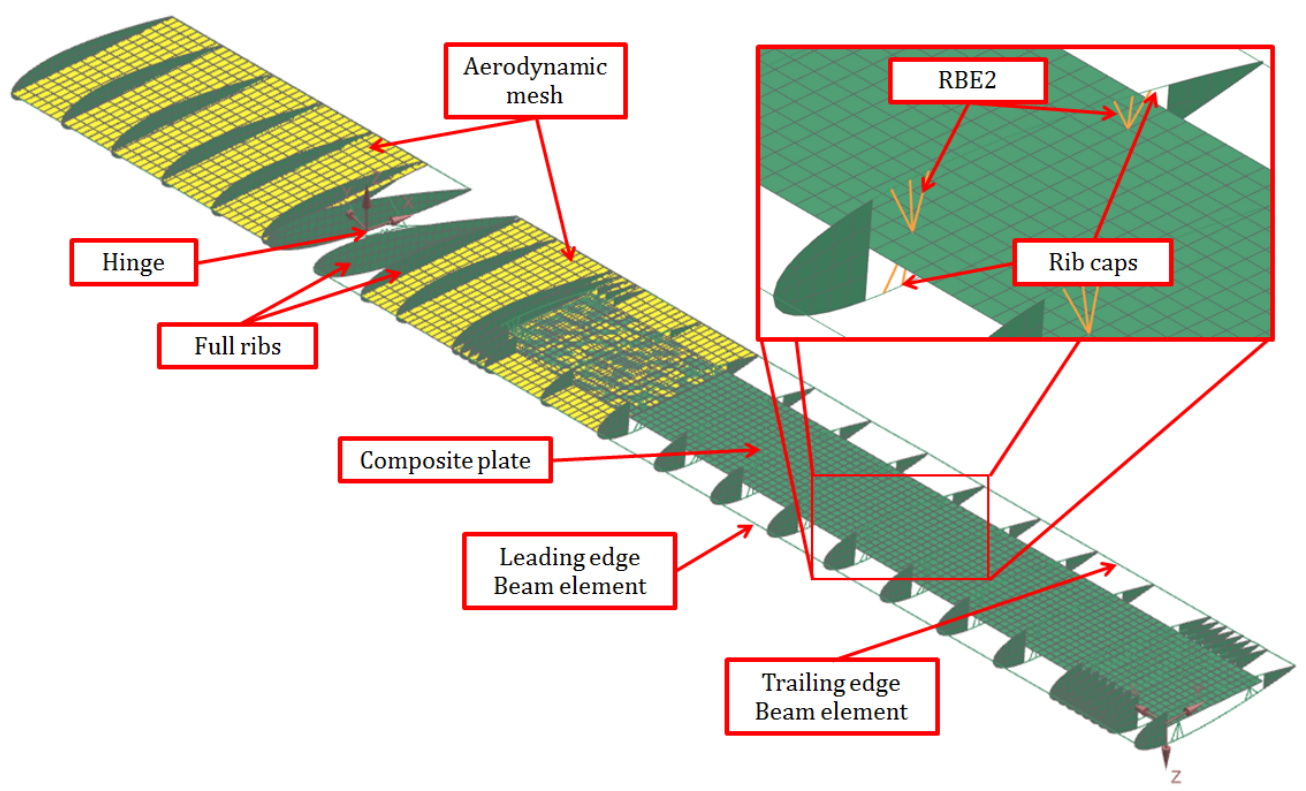

Fig. 3 FE model of the wing with a detail of the rib and the glue connection to the plate. Structural elements represented in green and aerodynamic mesh represented in yellow

The hinge is modeled following the approach proposed by Castrichini et al. [4]. The FE assembly of the hinge is shown in Figure 4. The hinge is modeled using elastic CBUSH elements. RBE2 elements connect the wing and wingtip to the axis nodes and the elastic elements connect both parts, as presented in Figure 4. Figure 4a shows the FEM representation and Figure 4b presents a sketch of the different connections. When the hinge is released, the rotational degree of freedom (DOF) aligned with the hinge spring should be set to zero stiffness, allowing a free rotation. However, this approach leads to a statically underdetermined structure and, ultimately, numerical divergence. To avoid this numerical problem, the stiffness of the rotational DOF is set relatively low stiffness, two orders of magnitude below the wing stiffness, hence making the spring act similarly to a mechanism. The remaining DOFs are set to relatively high stiffness values, two orders of magnitude above the wing stiffness, to model a rigid connection. On the other hand, when the wingtip is locked, the rotational DOF is also modeled as a rigid connection, hence limiting the rotation of the FWT.

\footnotetext{
"Simcenter 3D, Siemens, 2021. URL https://WWW.pIm.automation.siemens.com/global/en/products/simcenter/simcenter3d.html, retrieved 25 May 2021.

**MSc Nastran, MSc Software, 2021. URL https://www.mscsoftware.com/product/msc-nastran, retrieved 25 May 2021.
} 


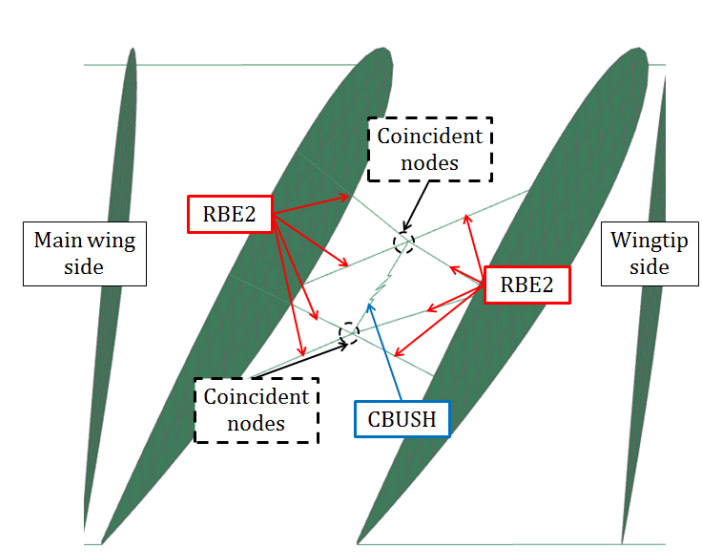

(a) Simcenter 3D FEM representation of the hinge

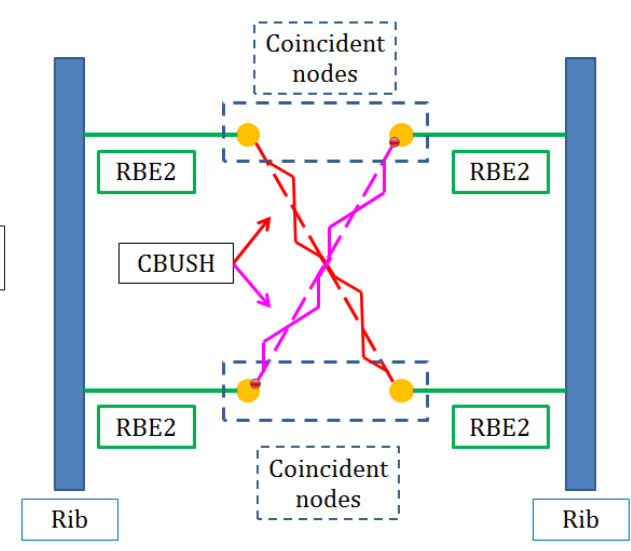

(b) Sketch of the connections used to model the hinge

Fig. 4 Details of the FEM used for the hinge

Finally, the aeroelastic model is completed by coupling the aerodynamic model to the structural model of the wing. Doublet Lattice Method (DLM) is used to determine the aerodynamic forces acting on the wing. As shown by Cheung et al. [5], DLM can only be applied to small deflections of the wingtip, hence only the time up to the hinge release is studied. Due to the wind tunnel setup, part of the wing has to be outside of the flow, so the aerodynamic mesh is only defined on the wingtip and the tip of the main wing, as seen in Figure 3.

\section{Wing Spar Design}

The wing spar in the form of a composite plate determines the main structural properties of the wing. For this study, five different plates are defined. The first three are designed with different bending stiffness to investigate how wing flexibility affects the load alleviation performance of the FWT. Using one of these plates as a reference, two additional plates are designed with similar bending stiffness but including the bend-twist coupling. The tailored plates are designed to include a wash-in and wash-out response, load enhancing, and load alleviation respectively, so the interaction between different aeroelastic tailoring objectives and the FWT can be studied.

Special balanced laminates are used to vary the bending stiffness of the first three wings. This stacking sequence is antisymmetric about the mid-plane and, each half is symmetric about its own mid-plane (1/4 plane and 3/4 plane respectively). This combination omits the bend-twist couplings and produces a quasi-isotropic behavior [I3]. Therefore, only the bending stiffness of the plates can be varied by changing the laminate thickness, which is done by adding $[0,90]_{\mathrm{s}}$ layers in the middle plane. As a consequence of this addition, the in-plane quasi-isotropic behavior is lost, but the out-of-plane quasi-isotropy is conserved. Wings featuring these plates are named A to C, in increasing stiffness order. The different thicknesses are selected taking into account the deflections obtained from the numerical model to avoid the FWT to move out of the wind tunnel section.

On the other hand, symmetric-only laminates that include the bend-twist coupling are used in the tailored wings. The stacking sequence of these laminates is optimized to maximize the bend-twist term of the compliance $a b d$ matrix, $d_{16}$, while keeping the bending compliance term, $d_{11}$, within $\pm 2 \%$ of the reference plate value. Wing B is used as a reference wing, while the wings featuring wash-in and wash-out composite tailoring are designated $\mathrm{B}_{\mathrm{WI}}$ and $\mathrm{B}_{\mathrm{WO}}$, respectively.

According to classical laminate plate theory (CLPT) [13] and using the reference system introduced in [Figure 1, for a pure bending load, $M_{x}$, a non-zero $d_{16}$ term would produce a rotation, $K_{S}$, around the longitudinal axis of the plate, $x$. Thus, maximizing or minimizing this term maximizes the wash-in or wash-out response, respectively.

The optimization process computes the compliance matrix making use of CLPT using each of the plies as design variables. The total number of plies in the laminate, $2 n$, is the same as the reference laminate used in wing B and the sequence is defined as symmetric. Due to this symmetry, the number of design variables is halved to $n$ plies, and the $b$ matrix becomes zero, eliminating the in-plane/out-of-plane couplings. The main constrain of the problem is to achieve bending compliance terms similar to the reference value from wing $\mathrm{B}$, hence the relative difference between the bending compliance terms is constrained to $\varepsilon_{d_{11}}$, equivalent to a $2 \%$ difference. In addition, the extension-shear 
coupling terms of the $a$ matrix, $a_{16}$ and $a_{26}$, are constrained to a small value, $\varepsilon_{a_{16}}$ and $\varepsilon_{a_{26}}$ respectively, to maintain similarity with respect to the reference plate. Furthermore, the design space is reduced to ply angles from $-90^{\circ}$ to $90^{\circ}$ in steps of $15^{\circ}$ to simplify the manufacturing process and limit the possible outcome of the optimization.

Given the simplicity of the model and the high number of design variables, the problem is solved using the genetic algorithm (GA) included in Matlab's Optimization library. The GA depends on the initial conditions and randomization of each generation, therefore, the optimization problem is repeated 2000 times with randomized initial conditions to estimate the global optimum and create a set of possible laminates, which are then selected for a subsequent sensitivity analysis. Notice also that, thanks to the symmetry condition, rotating the plate $180^{\circ}$ about the longitudinal axis of the wing produces the same bending properties but coupling properties changed in sign. Thus, the optimization process can be carried out once for the wash-in design and rotate the plate about the symmetry plane to create the wash-out design. The optimization problem is presented in Equation 1.

$$
\begin{gathered}
\underset{x}{\operatorname{minimize}} d_{16} \\
\text { subject to }\left|\frac{d_{11}}{d_{11, \text { ref }}}-1\right|<\varepsilon_{d_{11}}=0.02 \\
\left|a_{16}\right|<\varepsilon_{a_{16}}=10^{-9} \\
\left|a_{26}\right|<\varepsilon_{a_{26}}=10^{-9}
\end{gathered}
$$

Since the optimization yields several designs of similar performance, a sensitivity analysis is carried out on the obtained stacking sequences to assess the effect of manufacturing imperfections on the design and select the most robust design. The sensitivity analysis focuses on the variation of the ply angle since ply angle misalignment due to hand layup is considered to be the main manufacturing imperfection. The effects of these misalignments are studied by recomputing the compliance matrix of the best five results of the optimization but including random misalignments in their plies. Each ply, at a nominal angle $\theta_{i}$, includes a misalignment, $\gamma_{i}$, following a normal distribution such that $95 \%$ of the results are within $\pm 5^{\circ}$, representing the error that can be introduced with the manual lamination. These new laminates are defined by Equation 2.

$$
\begin{aligned}
& {\left[\theta_{1}+\gamma_{1}, \ldots, \theta_{i}+\gamma_{i}, \ldots \theta_{n}+\gamma_{n}\right]_{s}} \\
& \gamma_{i} \sim N\left(\mu=0^{\circ}, \sigma=2.5^{\circ}\right)
\end{aligned} \text { for } i=1,2, \ldots, n
$$

Such a perturbation of the selected laminates is repeated 5000 times to obtain a sensitivity envelope of the selected laminates to such random changes. The objective is to find the plate that keeps the compliance matrix bending component, $d_{11}$, as similar as possible to the reference plate. This component follows a normal distribution centered on the value of the design without the random deviations, $d_{11,0}$, as shown in Equation 3. Thus, the most robust design is that with the lowest deviation, $\sigma$.

$$
d_{11} \sim N\left(\mu=d_{11,0}, \sigma\right)
$$

To conclude, the results of this selection process are presented in Table 2. Furthermore, Figure 5 presents the engineering modulus of elasticity, $\tilde{E}_{11}(\theta)$, and the bending modulus of elasticity, $\tilde{D}_{11}(\theta)$, computed from the $a b d$ matrix as presented in Equation 4 [14]. This comparison shows the variation in bending stiffness between plates A to $\mathrm{C}$ and the variation in directional properties between the tailored plates, $\mathrm{B}_{\mathrm{WI}}$ and $\mathrm{B}_{\mathrm{WO}}$, and the reference plate, $\mathrm{B}$. The properties in the longitudinal axis of the plate, $\theta=0^{\circ}$, are presented in Table 3 .

$$
\begin{gathered}
\tilde{E}_{11}(\theta)=\frac{1}{a_{11}(\theta)} ; \tilde{D}_{11}(\theta)=\frac{1}{d_{11}(\theta)} \\
a(\theta)=T^{T} a T ; d(\theta)=T^{T} d T
\end{gathered}
$$

where:

$$
T=\left(\begin{array}{ccc}
\cos ^{2}(\theta) & \sin ^{2}(\theta) & 2 \cos (\theta) \sin (\theta) \\
\sin ^{2}(\theta) & \cos ^{2}(\theta) & -2 \cos (\theta) \sin (\theta) \\
-\cos (\theta) \sin (\theta) & \cos (\theta) \sin (\theta) & \cos ^{2}(\theta)-\sin ^{2}(\theta)
\end{array}\right)
$$


Table 2 Stacking sequence of the wing core plates. Defined using reference system from Figure 1.

\begin{tabular}{cc}
\hline \hline Plate & Stacking sequence \\
\hline A & {$[0,45,90,-45]_{s}[0,90,90,0]_{s}[0,-45,90,45]_{s}$} \\
B & {$\left[[0,45,90,-45]_{2}\right]_{s}\left[[0,-45,90,+45]_{2}\right]_{s}$} \\
C & {$\left[[0,45,90,-45]_{2}\right]_{s}[0,90,90,0]_{s}\left[[0,-45,90,+45]_{2}\right]_{s}$} \\
B $_{\mathrm{WI}}$ & {$[-30,-15,-45,-15,-15,75,-30,30,90,-75,30,15,-75,45,45,15]_{s}$} \\
$\mathrm{~B}_{\mathrm{WO}}$ & {$[30,15,45,15,15,-75,30,-30,90,75,-30,-15,75,-45,-45,-15]_{s}$} \\
\hline \hline
\end{tabular}
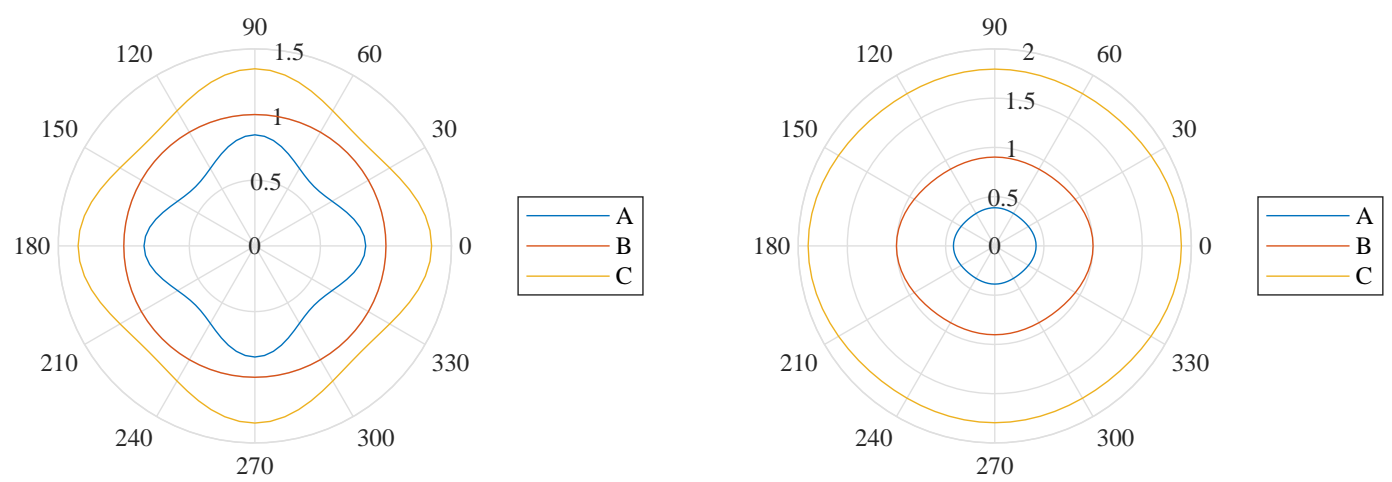

(a) Normalized engineering modulus of elasticity of (b) Normalized engineering bending modulus of plates plates with different bending stiffness

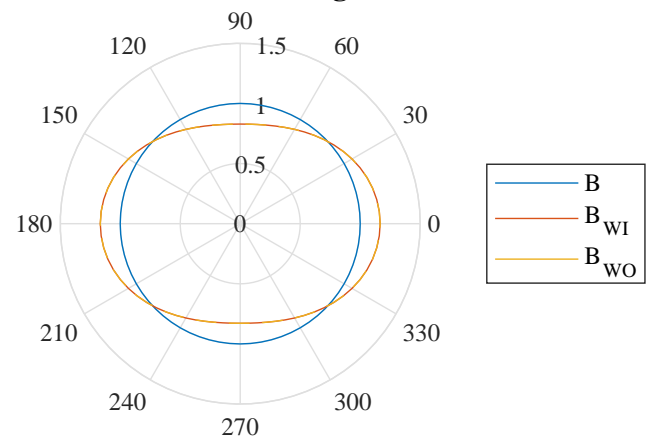
with different bending stiffness

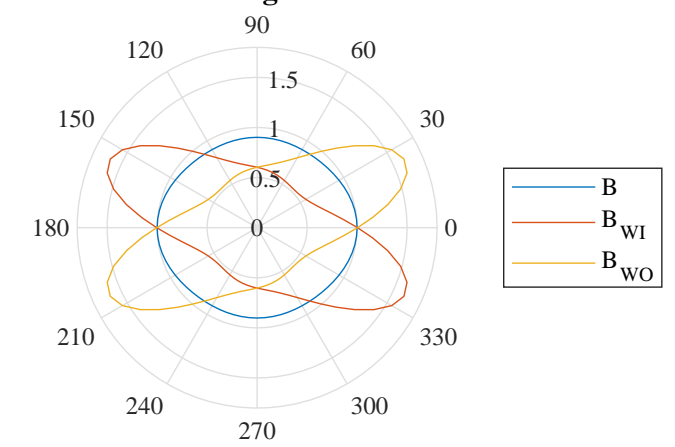

(c) Normalized engineering modulus of elasticity of (d) Normalized engineering bending modulus of plates plates with different tailoring with different tailoring

Fig. 5 Comparison of $\tilde{E}_{11}(\theta)$ and $\tilde{D}_{11}(\theta)$. Parameters normalized with maximum value of reference plate, B.

Table 3 Overview of bending compliance and coupling terms of the wing core plates

\begin{tabular}{ccccc}
\hline \hline Plate & $d_{11}[1 / \mathrm{Nmm}]$ & Thickness [mm] & Coupling & $d_{16}[1 / \mathrm{Nmm}]$ \\
\hline $\mathrm{A}$ & $1.82 \cdot 10^{-4}$ & 1.2 & - & 0 \\
$\mathrm{~B}$ & $7.67 \cdot 10^{-5}$ & 1.6 & - & 0 \\
$\mathrm{C}$ & $4.04 \cdot 10^{-5}$ & 2.0 & - & 0 \\
$\mathrm{~B}_{\mathrm{WI}}$ & $7.64 \cdot 10^{-5}$ & 1.6 & Wash-in & $6.19 \cdot 10^{-5}$ \\
$\mathrm{~B}_{\mathrm{WO}}$ & $7.64 \cdot 10^{-5}$ & 1.6 & Wash-out & $-6.19 \cdot 10^{-5}$ \\
\hline \hline
\end{tabular}

\section{Numerical simulations}

First, the normal modes of the wing are computed using SOL 103 or as the first step of the flutter analysis of SOL 145. These modes are important to understand how the wing behaves and, in the flutter analysis, to see what is bringing 
the wing to failure. The natural frequencies of each of the wings obtained using SOL 103 are presented in Table 4. On the one hand, the increasing bending stiffness from wing $\mathrm{A}$ to wing $\mathrm{C}$ is clearly reflected also in the increasing natural frequencies. Furthermore, the tailoring seems to increase the natural frequencies with respect to reference wing $\mathrm{B}$. This is independent of the wash-in or wash-out tailoring, as both wings $\mathrm{B}_{\mathrm{WI}}$ and $\mathrm{B}_{\mathrm{WO}}$ present higher natural frequencies than wing B. On the other hand, when the hinge is free, the trends of the first mode are different from those of the second. The second mode follows the same trends as for the locked-hinge condition while the first one does not seem to be affected by the structural properties of the wings.

The reason for this behavior change can be found in the shapes of these modes, which are presented in Figure 6. It can be seen how both modes in the locked-hinge condition are bending modes affecting the whole wing. However, the first mode in the free-hinge condition is a mode dominated by wing folding, referred to as a flapping mode. Since this mode only affects the FWT, whose properties do not differ between wings, the first flapping natural frequency is not affected by the main wing design. In addition, the second flapping mode is a combination of wing bending and FWT folding, hence also affected by the main wing stiffness properties.

Table 4 Comparison between wings of natural frequencies of first modes of free hinge (flapping) and locked hinge (locked) conditions (SOL 103/SOL 145).

\begin{tabular}{cccccc}
\hline \hline Mode & $\mathrm{A}$ & $\mathrm{B}$ & $\mathrm{C}$ & $\mathrm{B}_{\mathrm{WI}}$ & $\mathrm{B}_{\mathrm{WO}}$ \\
\hline Locked - 1st Bending [Hz] & 3.28 & 3.55 & 3.87 & 3.62 & 3.58 \\
Locked - 2nd Bending [Hz] & 18.17 & 18.87 & 19.55 & 18.96 & 19.01 \\
Free - 1st Flapping [Hz] & 1.40 & 1.41 & 1.42 & 1.41 & 1.41 \\
Free - 2nd Flapping [Hz] & 4.82 & 5.13 & 5.55 & 5.25 & 5.17 \\
\hline \hline
\end{tabular}

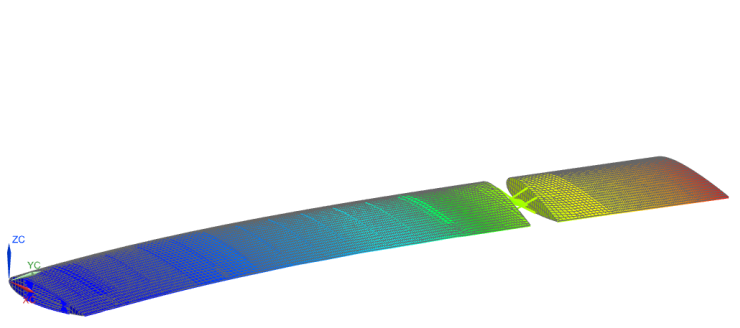

(a) Locked hinge, 1st Bending mode

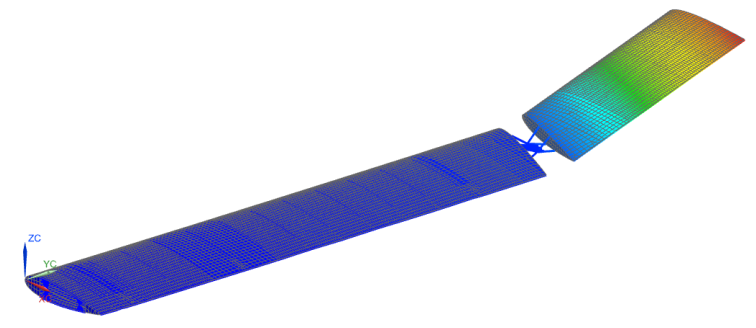

(c) Free hinge, 1st Flapping mode

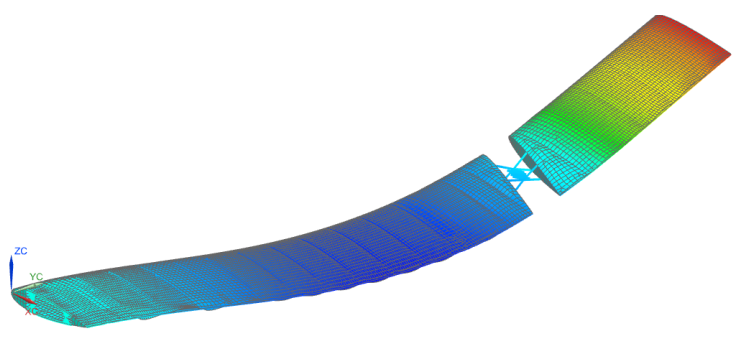

(b) Locked hinge, 2nd Bending mode

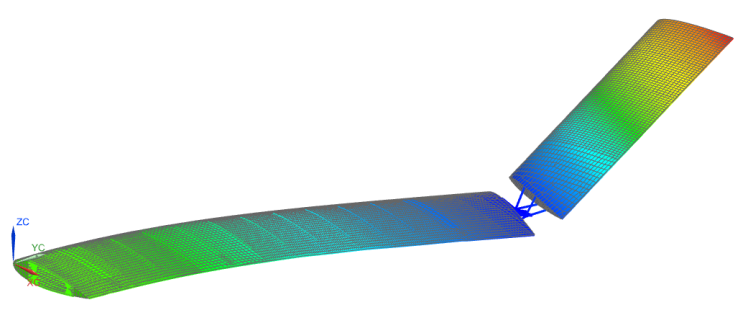

(d) Free hinge, 2nd Flapping mode

Fig. 6 Natural modes from simulation (SOL 103/SOL 145).

The results of the flutter analysis are summarized in पable 5. The bending stiffness of the wings increases the limiting speeds, as can be seen from the results of wings A, B, and C. In addition, this dependency on bending stiffness is more important when the hinge is locked. In the free-hinge condition, the flutter speed experiences a significant drop with respect to the locked-hinge condition. For a better understanding of this phenomena, the sensitivity of the flutter speed with respect to the changes in the hinge stiffness is investigated, as shown in Figure 7 by gradually increasing the hinge stiffness from 0 to the stiffness in the locked-hinge condition. A clear jump in flutter speed occurs between hinge stiffness of $20 \mathrm{Nmm} / \mathrm{rad}$ and $40 \mathrm{Nmm} / \mathrm{rad}$. To explain the reason for this jump, Figure 8 presents the damping and frequency diagrams for the cases close to the sudden variation. It can be seen how, for lower stiffness, the flutter 
Table 5 Instability speed comparison between wings in free-hinge and locked-hinge conditions (SOL 145).

\begin{tabular}{cccccc}
\hline Wing & $\mathrm{A}$ & $\mathrm{B}$ & $\mathrm{C}$ & $\mathrm{B}_{\mathrm{WI}}$ & $\mathrm{B}_{\mathrm{WO}}$ \\
\hline Free hinge $[\mathrm{m} / \mathrm{s}]$ & 19.9 & 21.1 & 23.0 & 20.4 & 22.9 \\
Locked hinge $[\mathrm{m} / \mathrm{s}]$ & 67.5 & 81.4 & 93.0 & 56.0 & 81.3 \\
\hline \hline
\end{tabular}

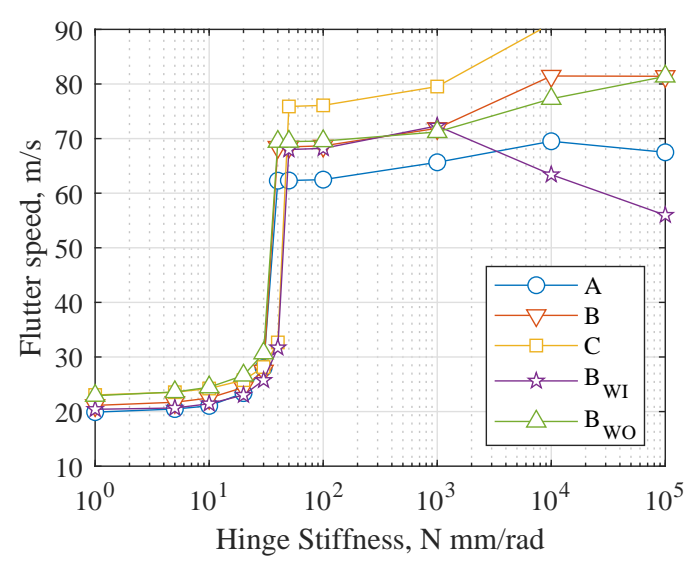

Fig. 7 Sensitivity analysis of flutter speed to hinge stiffness for the different wings.

mechanism is the second mode, while for the increased stiffness, flutter appears in the fourth mode. Thus, the speed drop between conditions is caused by a change in the flutter mechanism that drives the instability.

Finally, the flutter analysis also shows interesting behavior with respect to the wing tailoring and hinge-free and hinge-locked condition. Considering the reference studies on aeroelastic tailoring presented by Weisshaar [15], it is expected that the wash-in tailoring delays flutter and promotes divergence while the wash-out tailoring has an opposite effect. To explain the behavior in the locked-hinge condition, which would correspond to a conventional wing, Figure 9 presents a comparison of the damping and frequency flutter plots of wings $\mathrm{B}, \mathrm{B}_{\mathrm{WI}}$ and $\mathrm{B}_{\mathrm{WO}}$. For wing $\mathrm{B}_{\mathrm{WI}}$, there is a sudden drop in damping and frequency of the first mode, leading to a divergence instability. In contrast, the limiting aeroelastic instability for wings $\mathrm{B}$ and $\mathrm{B}_{\mathrm{WO}}$ is flutter. Nevertheless, when comparing the flutter speed of the tailored wings, the flutter speed of the $B_{W I}$ is higher than the flutter speed of the $B_{W O}$. These observations are in agreement with the expectations [15] as wash-in tailoring promotes divergence and delays flutter, while wash-out tailoring promotes flutter and delays divergence.

On the other hand, in the free-hinge condition, in all the wings, the limiting instability is flutter. However, in this case, the flutter speed of the $\mathrm{B}_{\mathrm{WI}}$ is lower than that of the $\mathrm{B}_{\mathrm{WO}}$, which is contrary to the expectations [II5]. This finding could be explained by the change in the flutter mechanism seen in the sensitivity analysis, hence the design rules applied for conventional aircraft wings might not be applicable for the FWT.

To conclude the flutter analysis, the sensitivity of the instabilities to the hinge stiffness and the change in the flutter mechanism show the complexity of the structural response of the hinge, and the drop in flutter speeds presents a possible limitation of the FWT. Keeping this in mind, the experiment is designed at an airspeed of $15 \mathrm{~m} / \mathrm{s}$ to avoid unexpected instabilities.

Next, static aeroelastic simulations using SOL 144 are used to estimate the steady-state loads and deflections of the wing. These simulations include gravity and study the wing at $\alpha=0^{\circ}$ and $\alpha=8^{\circ}$. On the one hand, since the airfoil used is symmetric and given the assumptions of the aerodynamic model, the first condition is equivalent to a static simulation in which the model is only loaded with gravity. On the other hand, the $\alpha=8^{\circ}$ condition introduces an aerodynamic load to compare it with the magnitude of the weight and study which force is dominant. To make it as similar as possible to real flight conditions, it is desired to have a system that is dominated by the aerodynamic force, so the 3D printed part is optimized to reduce its weight using these simulations as reference.

Finally, the gust response is studied with SOL 146. As explained by Cheung et al. [5], it cannot be used to solve gusts hitting the wing at a given static condition, i.e. $\alpha \neq 0^{\circ}$, so the problem is solved as a superposition of the static condition and the gust. First, the mesh is pre-deformed according to the results of the static analysis, and, afterward, 

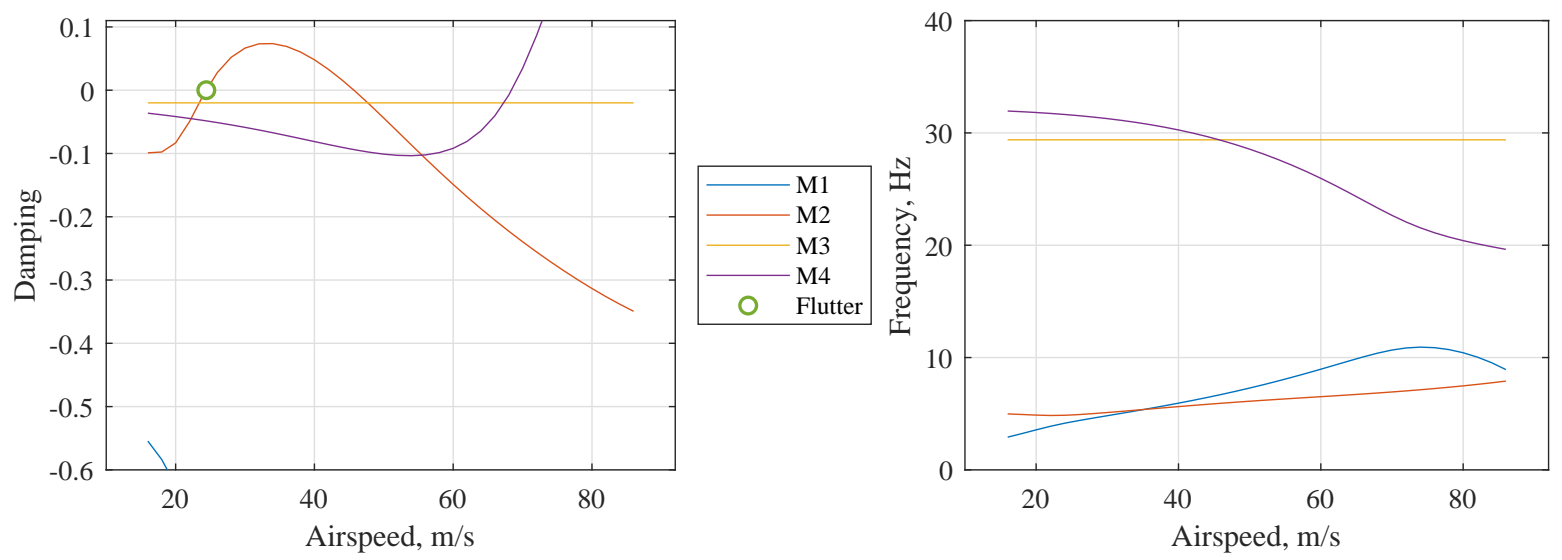

(a) $K_{\beta}=20 \mathrm{~N} \cdot \mathrm{mm} / \mathrm{rad}$
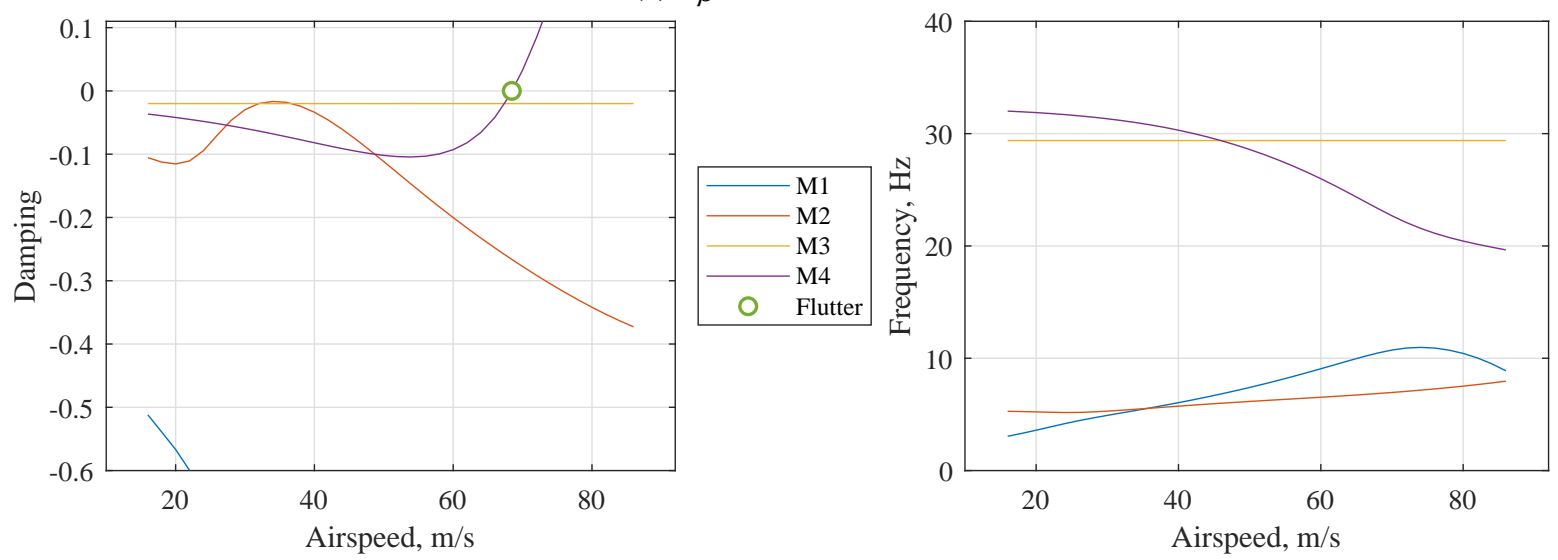

(b) $K_{\beta}=40 \mathrm{~N} \cdot \mathrm{mm} / \mathrm{rad}$

Fig. 8 Comparison of damping and frequency flutter plots of Wing B at different hinge stiffnesses, $K_{\beta}$.

the gust is simulated. The final result is then the sum of both. Notice that, given the linearity of the model, the validity of the results is limited to low fold angles, $\beta$. For this reason, the locked hinge condition is considered as an indicator of the maximum load and deflections but the free hinge condition is only considered qualitatively to understand its effects. The hinge release based on a load threshold cannot be simulated with the available tools, hence it is not considered.

These results are used to ensure the wing will remain in the wind tunnel section during the experiment and that the different designs offer a noticeable difference in their response. Figure 10 shows an example of comparison between the locked and free hinge conditions for two different gusts and Figure 11] shows a comparison of peak loads between wings at different gust frequencies.

From the individual gust responses, it is possible to see that the peak loads are similar but the time response is not. On the one hand, for the slow gust of $1.25 \mathrm{~Hz}$, the loads smoothly follow the gust and present a small oscillation after the gust when the hinge is locked. On the other hand, for the fast gust of $7.5 \mathrm{~Hz}$, there is a clear difference between the free-hinge and the locked-hinge conditions. While the oscillations disappear after a second for the locked hinge condition, the oscillations persist for twice the time in the free-hinge condition. This behavior is similar for all the wings, hence only these examples are presented.

Finally, in the peak load comparison, there is a clear difference between the magnitudes each wing can achieve. The peak loads decrease with the bending stiffness of the wings, hence wing A presents higher peak loads than wing B and wing $\mathrm{B}$ presents higher peak loads than wing $\mathrm{C}$. Furthermore, the tailoring also has a prominent effect: wing $\mathrm{B}_{\mathrm{WO}}$ presents the lowest peak loads, even lower than wing $\mathrm{C}$, while $\mathrm{B}_{\mathrm{WI}}$ shows higher peak loads than the reference wing $\mathrm{B}$, comparable to those from wing A. 

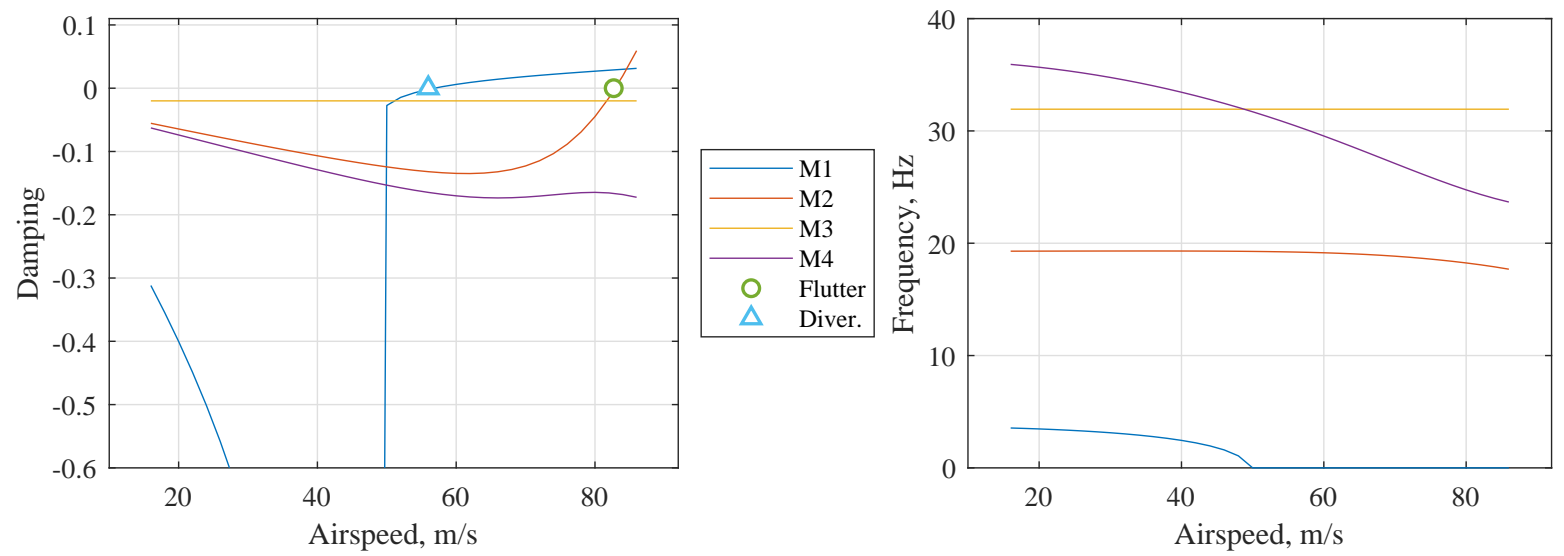

(a) Wing $\mathbf{B}_{\mathrm{WI}}$
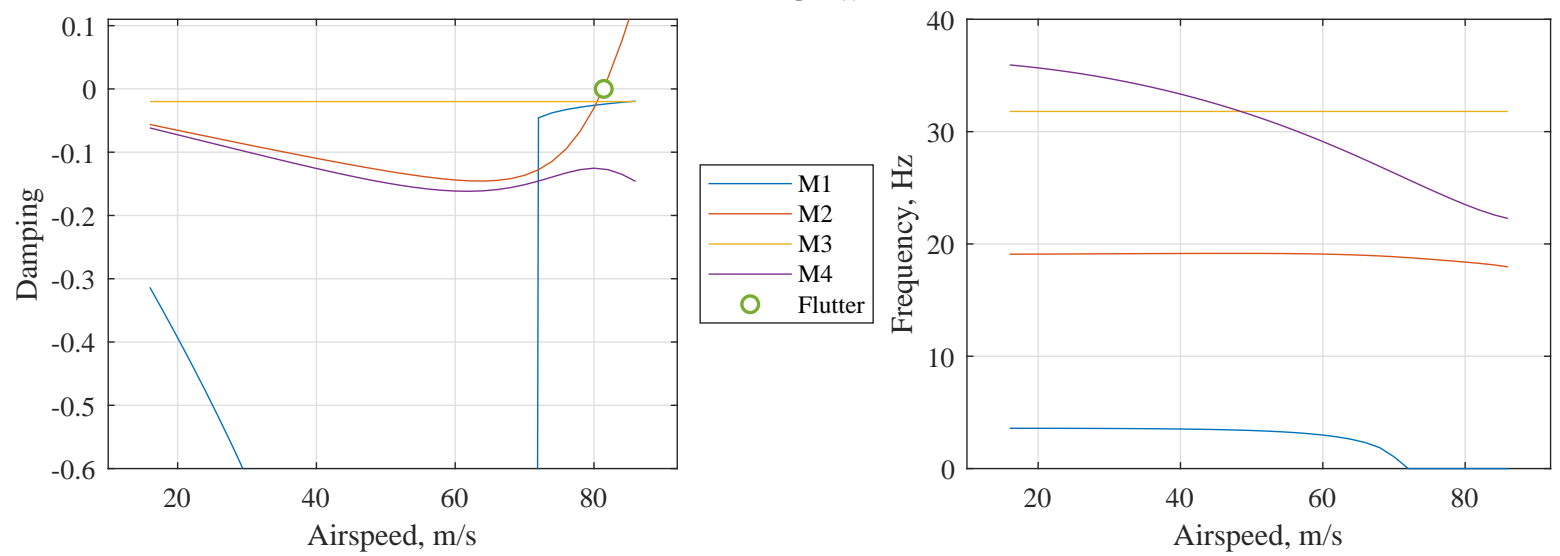

(b) Wing B
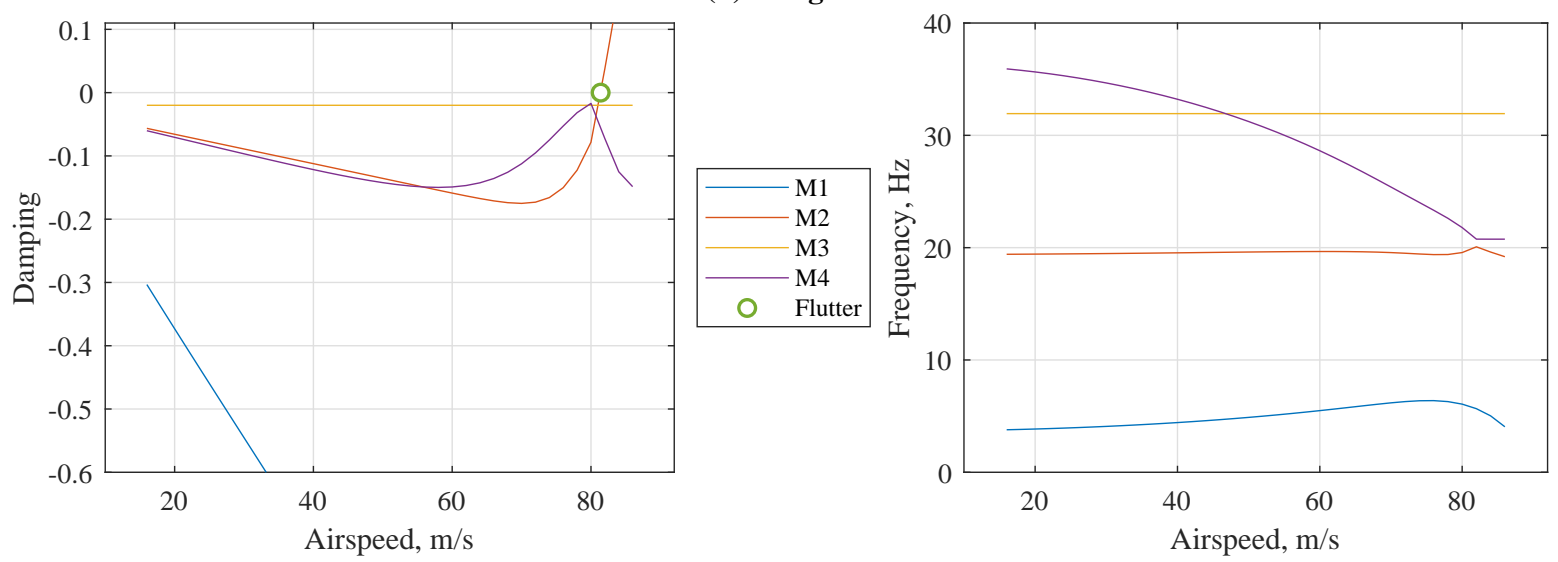

(c) Wing B BO

Fig. 9 Comparison of damping and frequency flutter plots between wings $B, B_{W I}$ and $B_{W o}$ in locked hinge condition.

III. Wind Tunnel Experiments

This section addresses procedure for the wind tunnel experiments, including the test setup, the sensors used, and the data acquisition procedure. Subsequently, the complete measurement procedure as well as data post-processing are described in more detail. 


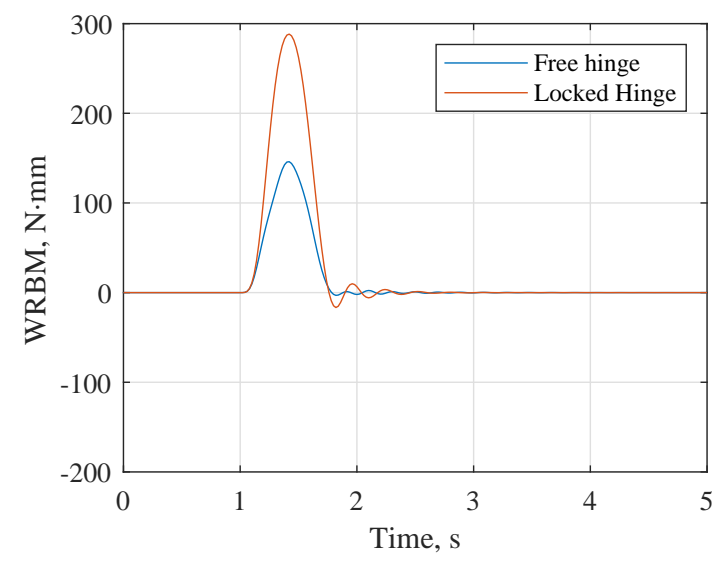

(a) Example of Wing $\mathrm{B}$ at $1.25 \mathrm{~Hz}$

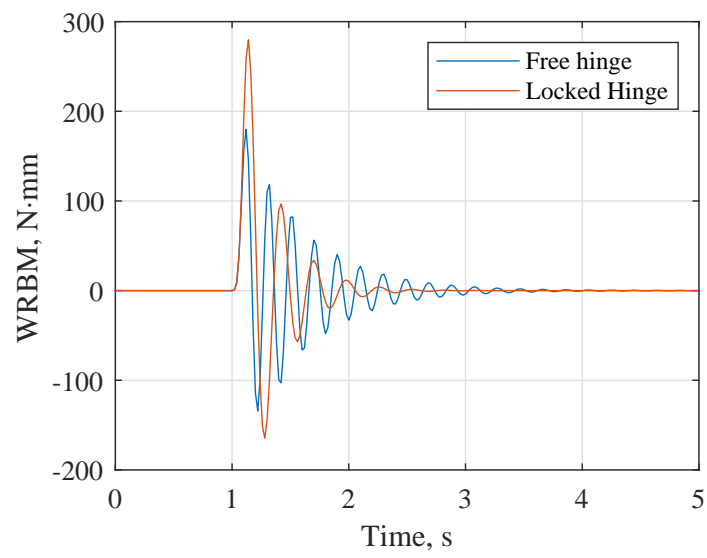

(b) Example of Wing $\mathrm{B}$ at $7.5 \mathrm{~Hz}$

Fig. 10 Results of gust simulations (SOL 146). Comparison between locked-hinge and free-hinge conditions for different gust frequencies.

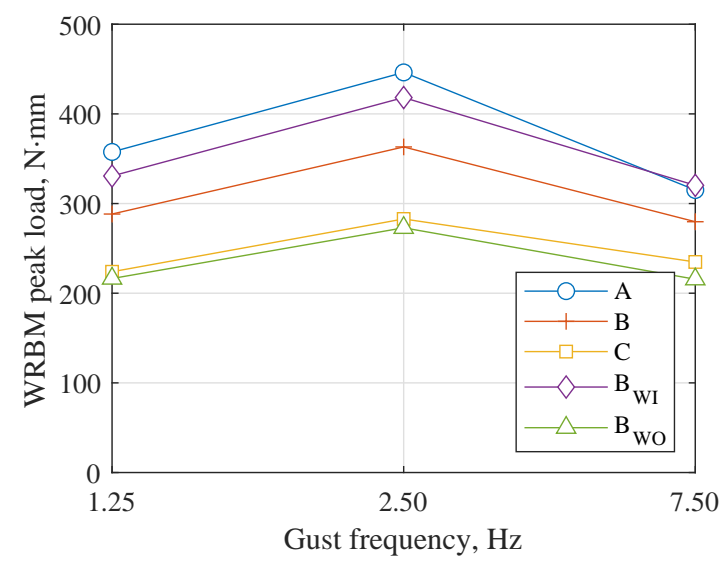

Fig. 11 Peak WRBM load comparison between plates for different gust frequencies in locked-hinge condition (SOL 146).

\section{A. Test Setup}

The experiments are conducted in the W-Tunnel at the Delft University of Technology, which is an open jet wind tunnel with a square test section of $0.4 \times 0.4 \mathrm{~m}^{2}$. The tests are performed at a free-stream velocity of $10 \mathrm{~m} / \mathrm{s}$, which has to be reduced with respect to the design free-stream velocity due to the occurrence of flutter, as further addressed in section $1 \mathrm{~V}$. A gust generator is mounted at the outlet of the tunnel nozzle to create the gust perturbations. An overview of the gust generator and the wing model is presented in Figure 12.

Figure 13 shows the sensors mounted on the wing. Two Kyowa KFGS-10-120-D17-16 triaxial $0^{\circ} / 90^{\circ} / 45^{\circ}$ strain gauge rosettes are used on the top and bottom faces of the plates to measure the deformation at the root of the wing, which is related to the WRBM. The central strain gauge, $45^{\circ}$, is positioned in the spanwise direction, leaving the $0^{\circ}$ and $90^{\circ}$ strain gauges at $\pm 45^{\circ}$ from the spanwise direction of the wing. Each of the top strain gauges is connected to the corresponding parallel strain gauge from the bottom rosette in half-bridge configuration. For example, the strain gauge from the top rosette at $45^{\circ}$ is connected to the strain gauge from the bottom rosette at $-45^{\circ}$, as the rosette is turned by $180^{\circ}$ around the spanwise direction. Secondly, two PCB 352A24 uniaxial accelerometers 7 are used to characterize the main wing tip displacement. Finally, the fold angle of the wingtip is measured with a Bourns 3310C-125-203L

\footnotetext{
${ }^{\dagger \dagger}$ KFGS Series, General-purpose Foil Strain Gages, KYOWA, 2021. URL https://wwW . kyowa-ei .com/eng/product/category/strain_ gages/kfgs/index.htmL, retrieved 28 July 2021.

${ }^{\ddagger}$ PCB 352A24 Uniaxial Accelerometer, PCB Piezotronics, 2021. URL https://wWW.pcb. com/products?mode1=352A24, retrieved 28 July 2021.
} 


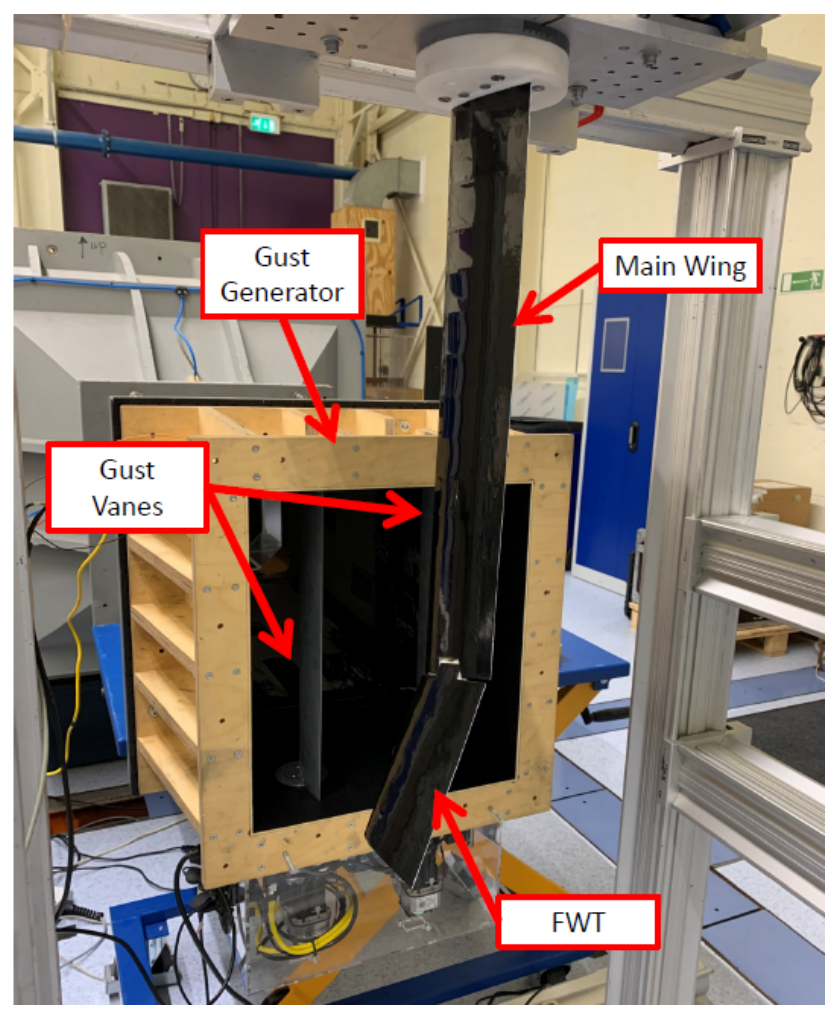

Fig. 12 Overview of the gust generator and test object.

potentiometer ${ }^{\text {[\$ }}$ connected to the shaft of the FWT.

The data acquisition is implemented by the system represented in the schematic in Figure 14. The gust generator creates a sequence of 1-cosine gusts with a fixed time interval. At the start of each gust, a trigger signal is generated and sent to both an Arduino UNO ${ }^{\mathbb{\Psi}}$ and a SCADAS Mobile when the trigger is received, the microcontroller waits for a specified time and then releases the mechanism; after a few seconds, the hinge is taken back to the locked position. The SCADAS is in charge of the data acquisition: it reads all the sensors, as well as the trigger signal from the gust generator and a control signal generated by the Arduino in order to keep track of the actuator motion. This signal is compared to the trigger and potentiometer signals to monitor the delays in the actuation chain. Finally, the SCADAS information is sent to a National Instruments cRIO-9744 using EtherCAT communication. The cRIO converts the electrical signals into the respective measurements making use of calibration constants and saves the data into the main computer. The whole setup is presented in Figure 15.

\section{B. Experimental procedure}

\section{Strain gauge calibration}

The strain gauge bridge requires a calibration to allow the determination of the loads from the bridge output. The linear transformation, $\bar{c}$, is a matrix that relates the bending loads, $M_{X}$, and torque loads, $M_{S}$, to the strain gauge bridge signals, $V$, as presented in Equation 6.

\footnotetext{
$\S \S$ Bourns 3310 series, Bourns, 2021. URL https://www.bourns.com/products/potentiometers/industrial-panel-controls/ product/3310, retrieved 28 July 2021.

IIII Arduino UNO \& Genuino UNO, Arduino, 2021. URL https://wwW . arduino . cc/en/pmw1k1 . php?n=Main/arduinoBoardUno, retrieved 28 July 2021.

*** Simcenter SCADAS Mobile and SCADAS Recorder, Siemens - Community support, 2021. URL https://community. Sw.S1emens.com/ s/article/simcenter-scadas-mobile-and-scadas-recorder, retrieved 28 July 2021.

$\dagger \dagger$ cRIO-9074, National Instruments - Support, 2021. URL https://wWW.n1.com/nl-nl/support/mode1.crio-9074.htm1, retrieved 28 July 2021.
} 


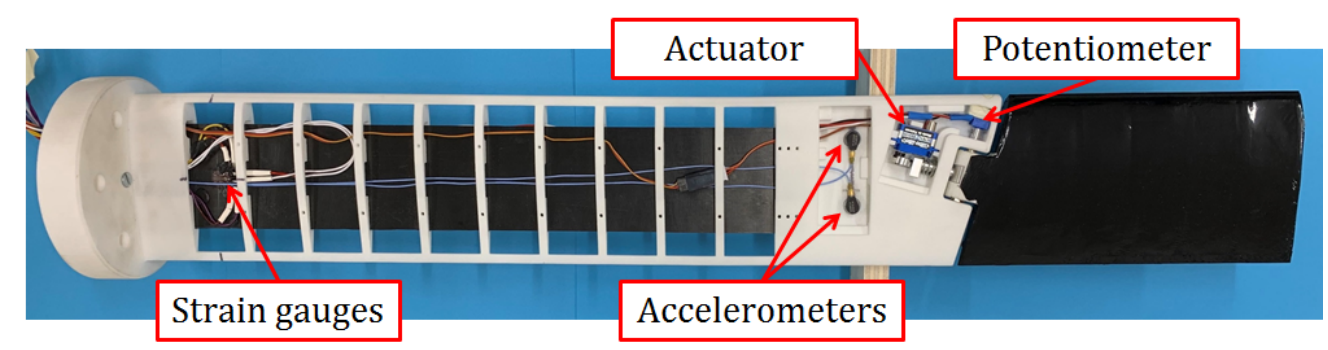

Fig. 13 Distribution of sensors along the wing

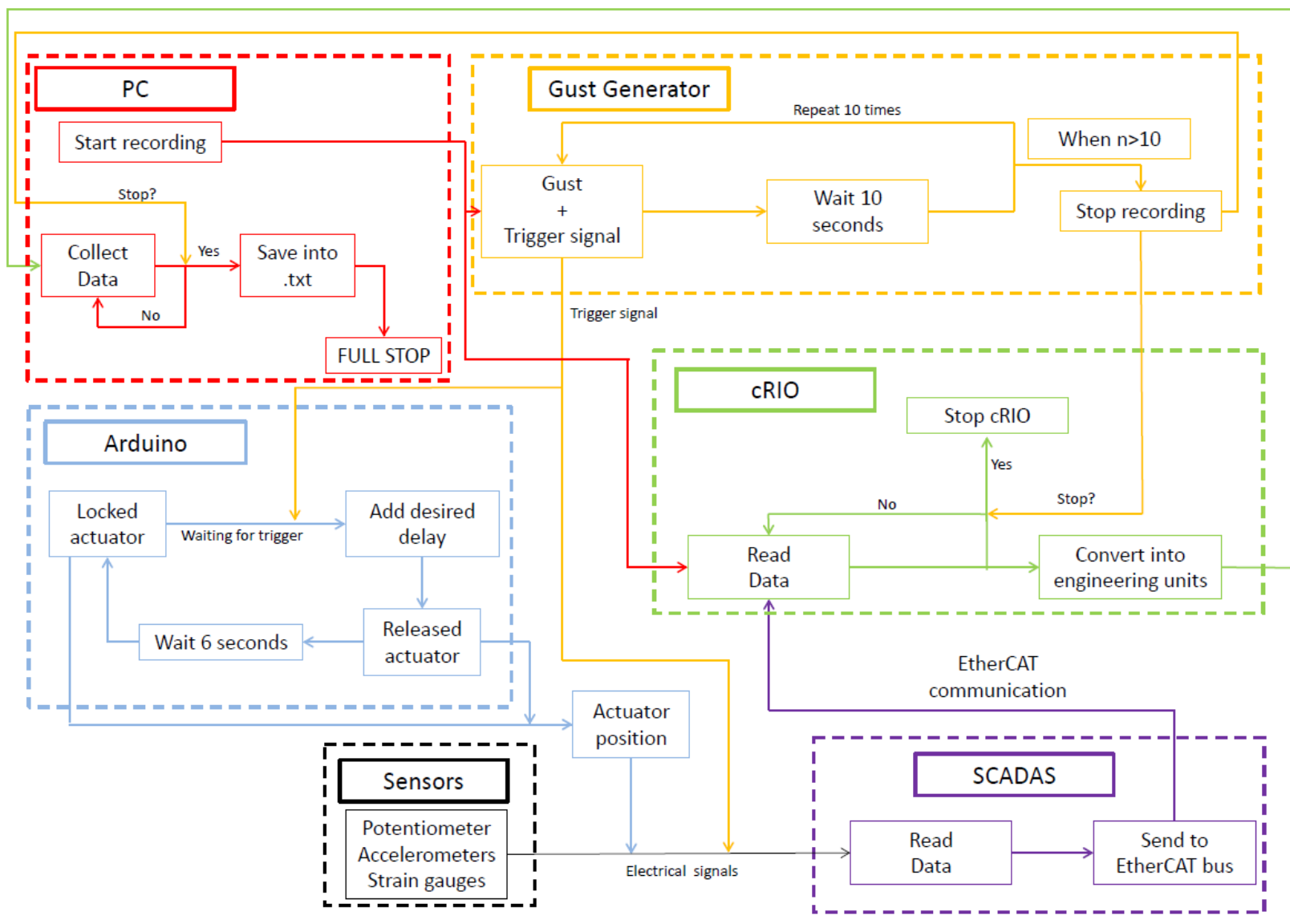

Fig. 14 Schematic of the data acquisition and control system

$$
\left(\begin{array}{c}
V_{+45} \\
V_{0} \\
V_{-45}
\end{array}\right)=\overline{\boldsymbol{c}}\left(\begin{array}{l}
M_{X} \\
M_{S}
\end{array}\right)=\left(\begin{array}{ll}
c_{1,1} & c_{1,2} \\
c_{2,1} & c_{2,2} \\
c_{3,1} & c_{3,2}
\end{array}\right)\left(\begin{array}{c}
M_{X} \\
M_{S}
\end{array}\right)
$$

Applying pure bending and torque loads separately, it is possible to obtain the calibration constants, $c_{i, j}$, using the least-squares (LS) solution according to Equation 7, where $\boldsymbol{M}_{\boldsymbol{j}}$ and $\boldsymbol{V}_{\boldsymbol{i}}$ are vectors of known loads and the signal of the strain gauge in $i$ direction, respectively.

$$
\boldsymbol{V}_{\boldsymbol{i}}=c_{i, j} \cdot \boldsymbol{M}_{\boldsymbol{j}} \rightarrow c_{i, j}=\left(\boldsymbol{M}_{\boldsymbol{j}}^{\boldsymbol{T}} \boldsymbol{M}_{\boldsymbol{j}}\right)^{-1} \boldsymbol{M}_{\boldsymbol{j}}^{\boldsymbol{T}} \boldsymbol{V}_{\boldsymbol{i}}
$$

Once the calibration constants are known, the inverse transformation, $\bar{C}$, can be computed using the pseudo-inverse 


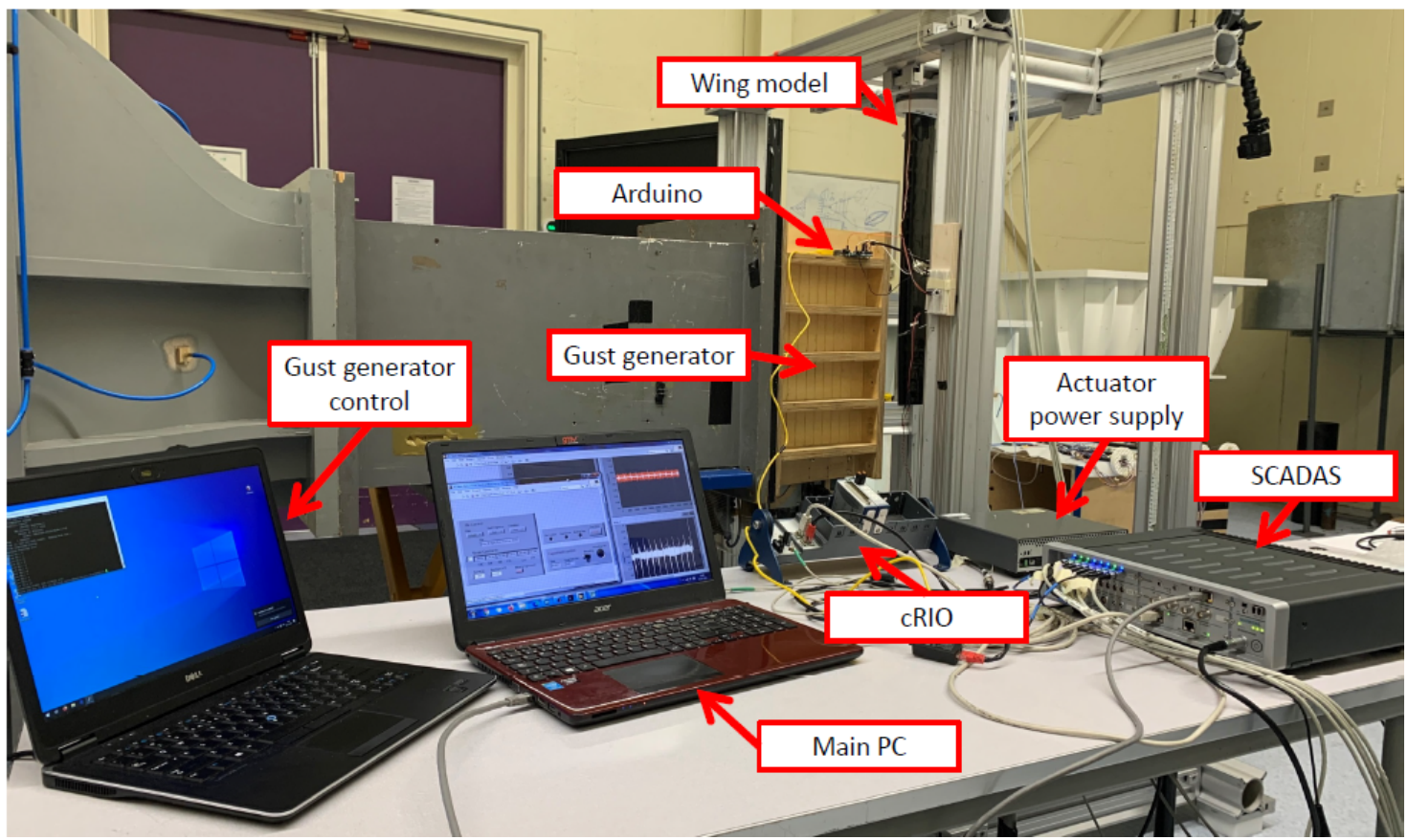

Fig. 15 Wind tunnel setup

concept already used in the LS solution:

$$
V=\bar{c} M \rightarrow\left(\bar{c}^{T} \bar{c}\right)^{-1} \bar{c}^{T} V=M \rightarrow M=\bar{C} V
$$

Finally, this calibration is validated by applying combinations of bending and torsion loads and comparing them to the loads obtained after transforming the strain gauge signals with the calibration matrix. As shown in Table 6 , the maximum error for the bending moment is below 10\%. However, the error in the torque measurement can reach up to $80 \%$ and is very inconsistent between wings. These differences could be caused by the cross-measurement of the $\pm 45^{\circ}$ strain gauges since these strain gauges are affected by both bending and torque loads, which is not the case for the central strain gauge oriented along the span which is only affected by bending loads. Since the bending loads are higher than the torque, the measurements of these strain gauges are dominated by bending, which affects the calibration matrix. In view of this limitation, the torque is not considered in the results.

Table 6 Comparison of maximum relative error between the calibrated measured load and the applied load

\begin{tabular}{cccccc}
\hline \hline Wing & A & B & C & B In & B Out \\
\hline Bending [\%] & 5.5 & -7.7 & 6.8 & -6.5 & -3.6 \\
Torque [\%] & 81.2 & 14.4 & -23.8 & 36.7 & 35.9 \\
\hline \hline
\end{tabular}

\section{Test procedure}

The experiment is carried out for each of the wings presented in Table 3. The investigation starts with characterizing the static behavior of the wings by changing the angle of attack, $\alpha$, between $-6^{\circ}$ and $14^{\circ}$. These measurements are collected in polar curves of the bending moment coefficient, $C_{B}$, defined by Equation 9, and the fold angle, $\beta$.

$$
C_{B}=\frac{M_{X}}{\frac{1}{2} \rho S B U^{2}}
$$

Next, the time response of the wings to a 1-cosine gust excitation is recorded for different angles of attack, gust frequencies, and release thresholds. The variation in the angle of attack separates a purely dynamic load $\left(\alpha=0^{\circ}\right)$ from 
a more representative combination of dynamic load and static load introduced by a non-zero angle of attack $\left(\alpha=5^{\circ}\right)$. The gust frequencies selected for the experiment are spread below, above, and close to the natural frequencies in free-hinge and locked-hinge conditions to cover a broad dynamic range. The first natural frequency in the locked-hinge configuration is referred to as 1st Bending, while the first one in the free-hinge condition is referred to as 1st Flapping. These natural frequencies are found with a modal impact test, which is performed in-situ when the wing is mounted in the wind tunnel.

Finally, the threshold variations include the free-hinge and locked-hinge conditions as a reference and different release conditions during the gust: i) released with the gust generator trigger signal before the gust hits the wing, ii) when the gust hits the wing, i.e. the load starts increasing, iii) it is released when the load achieves $50 \%$ of its peak load, and iv) at the peak load. These release conditions are referred to as pre-released, $0 \%, 50 \%$, and 100\% release, respectively. An overview of the test cases is presented in एable 7. For each specific dynamic testing condition, the experiment is repeated 10 times to allow for statistical evaluation of the measured aeroelastic responses.

Table 7 Overview of test cases

\begin{tabular}{ccc}
\hline \hline Parameter & Static Load & 1-cosine gust \\
\hline Plate & \multicolumn{2}{c}{$\mathrm{A}, \mathrm{B}, \mathrm{C}, \mathrm{B}_{\mathrm{WI}}, \mathrm{B}_{\mathrm{WO}}$} \\
Hinge condition & \multicolumn{2}{c}{ Free, Locked, Pre-released, 0\%, 50\%, 100\% } \\
$\alpha\left[^{\circ}\right]^{\circ}$ & {$[-6,14]$} & 0,5 \\
Gust amplitude $\left[^{\circ}\right]$ & - & 2.5 \\
Gust frequency $[\mathrm{Hz}]$ & - & $0.5,5,8,1$ st Bending, 1st Flapping \\
\hline \hline
\end{tabular}

\section{Measurement Post-processing}

The assessment of the results is carried out using two main parameters: peak loads and root mean square (RMS). Figure 16 shows an example of variation of the bending moment coefficient over time at $\alpha=5^{\circ}$. The peak load, $\Delta C_{B}$, is defined as the difference between the maximum load, $C_{B, \max }$, and the mean pre-release steady-state load, $C_{B, p r e}$, i.e. the static load before the gust hits the wing.

The RMS is used as an indicator of the persistence of the oscillations in the load signals. After the gust encounter, the dynamic load settles on the static load corresponding to the locked-hinge or free-hinge condition. Therefore, the oscillations that persist for a longer time result in a higher RMS value. Since there are two steady states corresponding to the locked-hinge and free-hinge conditions, as seen in Figure 16. The RMS is calculated using $\tilde{C}_{B}(t)$, as defined in Equation 10.

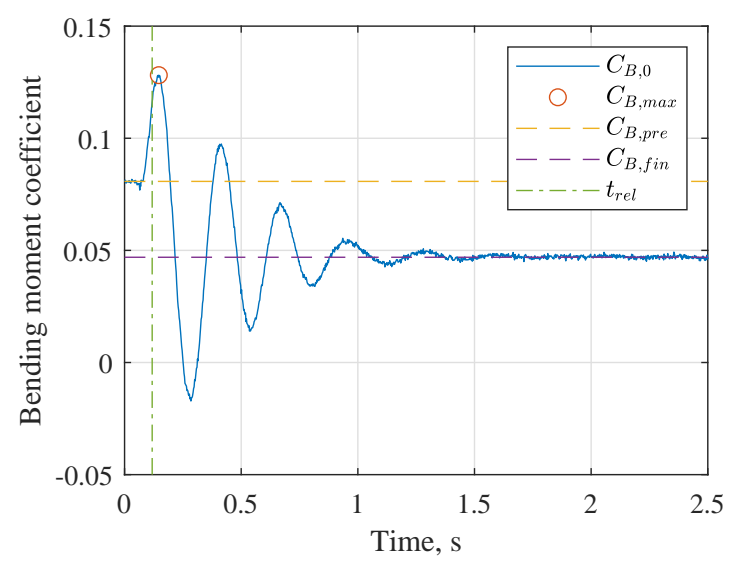

Fig. 16 Terminology used for processing the data 


$$
\tilde{C}_{B}(t)=\left\{\begin{array}{lc}
C_{B, 0}(t)-C_{B, p r e} & \text { for } 0<t<t_{r e l} \\
C_{B, 0}(t)-C_{B, \text { fin }} & \text { for } t>t_{\text {rel }}
\end{array}\right.
$$

\section{Results}

The experimental results are presented in this section and, where possible, compared with the numerical results. In the initial phase of the wind tunnel campaign, it was discovered that the actual flutter speed was considerably lower than expected. As a result, the free stream velocity had to be reduced from $15 \mathrm{~m} / \mathrm{s}$ to $10 \mathrm{~m} / \mathrm{s}$ to operate in safe, flutter-free conditions across all the wings. Consequently, it was decided to measure the wings in a vertical orientation to avoid their response dominated by weight rather than aeroelastic forces.

\section{A. Test Conditions: Flutter}

After the first design space exploration, a stability study is carried out for each wing. The airspeed is increased in steps of $1 \mathrm{~m} / \mathrm{s}$ and the wing is excited to observe its response. The wing is considered unstable when the transient response fails to die out. In Table 8, the simulated flutter speeds and measured flutter speeds are presented. Furthermore, the wings are also tested in the locked-hinge condition but do not show sustained or divergent instabilities below 25 $\mathrm{m} / \mathrm{s}$, the maximum operational speed of the wind tunnel.

Table 8 Comparison between experimental and simulated flutter speeds.

\begin{tabular}{cccccc}
\hline \hline Wing & A & B & C & B $_{\text {WI }}$ & B $_{\text {WO }}$ \\
\hline Simulation $[\mathrm{m} / \mathrm{s}]$ & 19.9 & 21.1 & 23.0 & 20.4 & 22.9 \\
Experiment $[\mathrm{m} / \mathrm{s}]$ & 12 & 16 & 17 & 15 & 18 \\
\hline \hline
\end{tabular}

It can be seen how the trends observed in the experimental results are the same observed in the simulations but at much lower speeds. The increase in the flutter speed across wings A, B, and C shows the expected result of bending stiffness delaying flutter. Considering the tailored wings, the flutter speed of the $\mathrm{B}_{\mathrm{WI}}$ wing is the lowest while the flutter speed of the $\mathrm{B}_{\mathrm{WO}}$ is the highest, which is in agreement with the observations presented in subsection - [I.B. In this regard, the experiments confirm that the FWT in a hinge-free condition can considerably alter the aeroelastic stability behavior of tailored composite wings.

In addition, the agreement between the trends seen in the experiment and those from the numerical results shows that the model offers a good representation of the aeroelastic problem but needs to be improved for more accurate results. Therefore, a ground vibration test (GVT) is planned as future work to correlate the numerical model with the experiment and update the stiffness and mass properties accordingly.

\section{B. Natural Modes: Modal Impact Test}

Table 9 compares the numerical and experimental results for the first natural frequencies of the tested wings in both locked-hinge and free-hinge conditions. It can be seen how the simulations follow similar trends as the experiments but add an offset, which is consistent between plates but changes between modes. This could be caused by an overprediction of the stiffness and a misprediction of the mass and center of gravity of the wing. Taking into account that the simulations rely on modal analysis for the flutter predictions, these discrepancies could explain the overprediction of flutter speed and a model update as suggested could improve the results.

Notice also that the 1st flapping frequency is the same for all the wings and the offset is much lower than for the other modes. Looking at the representation of the mode in the simulation, it can be seen that only the wingtip is affected, as shown in Figure 6c. This explains why the frequency is independent of the main structure properties and, the similarity in magnitude could mean that the wingtip properties have been modeled correctly.

Finally, the 1st Bending and 1st Flapping frequencies are used as one of the gust excitation frequencies during the gust experiments to study the effect on the GLA performance of exciting the wings at their natural frequencies. However, the gust generator can only produce gusts of frequencies from $0.5 \mathrm{~Hz}$ to $10 \mathrm{~Hz}$ in steps of $0.5 \mathrm{~Hz}$, therefore the excitation frequencies are rounded to the closest possible frequencies considering the results from the impact test. 
Table 9 Comparison of natural frequencies obtained from modal impact test and simulation

\begin{tabular}{|c|c|c|c|c|c|c|c|c|c|c|}
\hline \multirow{2}{*}{$\begin{array}{c}\text { Wing } \\
\text { Source }\end{array}$} & \multicolumn{2}{|r|}{ A } & \multicolumn{2}{|r|}{ B } & \multicolumn{2}{|r|}{$\mathrm{C}$} & \multicolumn{2}{|r|}{$\mathrm{B}_{\mathrm{WI}}$} & \multicolumn{2}{|r|}{$\mathrm{B}_{\mathrm{WO}}$} \\
\hline & Exp. & Sim. & Exp. & Sim. & Exp. & Sim. & Exp. & Sim. & Exp. & Sim. \\
\hline 1st Bending $[\mathrm{Hz}]$ & 2.76 & $3.28(+18.8 \%)$ & 3.03 & $3.55(+17.2 \%)$ & 3.42 & $3.87(+13.2 \%)$ & 3.23 & $3.62(+12.1 \%)$ & 3.23 & $3.58(+10.8 \%)$ \\
\hline 1st Flapping [Hz] & 1.33 & $1.40(+5.2 \%)$ & 1.33 & $1.41(+6.0 \%)$ & 1.33 & $1.42(+6.8 \%)$ & 1.33 & $1.41(+6.0 \%)$ & 1.33 & $1.41(+6.0 \%)$ \\
\hline 2nd Flapping [Hz] & 3.49 & $4.82(+38.1 \%)$ & 3.90 & $5.13(+31.5 \%)$ & 4.40 & $5.55(+26.1 \%)$ & 4.09 & $5.25(+28.4 \%)$ & 4.10 & $5.17(+26.1 \%)$ \\
\hline
\end{tabular}

As a result, the 1st Flapping frequency used in the experiment is $1.5 \mathrm{~Hz}$ for all the wings and the 1st Bending frequency is $3.0 \mathrm{~Hz}$ for all but wing $\mathrm{C}$, which is tested at $3.5 \mathrm{~Hz}$.

\section{Static Load: Bending Polar}

The moment coefficient polar is the first step to understanding the impact of the design variations on the exerted aerodynamic loads. Figure 17 shows the example of the bending moment coefficient and fold angle polars for wing B, which shows a behavior similar to the other wings. The load alleviation due to the released FWT can be clearly observed by the reduced slope of the bending moment coefficient line, which is in agreement with the observations presented by Cheung et al. [5]. Notice also that, for high angles of attack, stall causes the bending moment to decrease while the measurement deviations increase. The fold angle polar shows how the FWT rotates to keep the load equilibrium when the hinge is released. In addition, for the example of wing B when the hinge is locked, the measured angle is not zero but varies from $-1^{\circ}$ at the lowest angle of attack to $1^{\circ}$ at the highest. This could be caused by the compliance of the hinge locking mechanism, allowing some displacement of the FWT.

Table 10 presents a summary of the results of the different wings, including the polar slope and the stall angle. On the one hand, wings A, B, and C present the same locked slope and stall angles, which show that the results are not significantly affected by the bending stiffness. On the other hand, due to the added bend-twist coupling caused by the wash-out tailoring of wing $\mathrm{B}_{\mathrm{WO}}$, the slope decreases, and the stall is delayed. This behavior is reversed for $\mathrm{B}_{\mathrm{WI}}$,
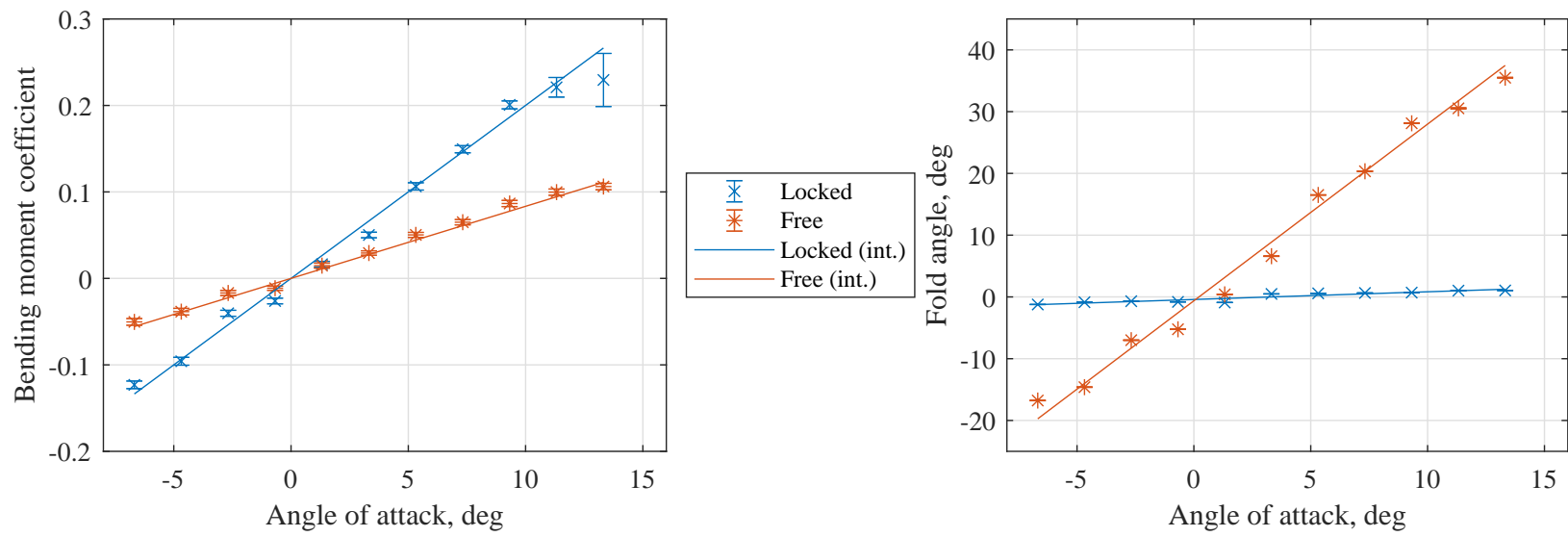

Fig. 17 Plate B interpolated $C_{B}$ and $\beta$ polars

Table 10 Slopes and stall angles obtained from $C_{B}$ polars. Slopes presented in counts $\left(C_{B}=0.0001=1\right.$ count $)$

\begin{tabular}{ccccc}
\hline \hline Wing & Locked $C_{B, \alpha}\left[1 /^{\circ}\right]$ & Stall angle $\left[{ }^{\circ}\right]$ & Free $C_{B, \alpha}\left[1 /^{\circ}\right]$ & Stall angle $\left[{ }^{\circ}\right]$ \\
\hline $\mathrm{A}$ & 200 & 13 & 89 & - \\
$\mathrm{B}$ & 200 & 13 & 83 & - \\
$\mathrm{C}$ & 199 & 13 & 85 & - \\
$\mathrm{B}_{\mathrm{WI}}$ & 204 & 11 & 73 & 15 \\
$\mathrm{~B}_{\mathrm{WO}}$ & 173 & 15 & 75 & - \\
\hline \hline
\end{tabular}


although the effect on the slope with respect to the reference wing, $\mathrm{B}$, is not as clear as for $\mathrm{B}_{\mathrm{WO}}$. The asymmetry in the response between the tailored wings could be caused by imperfections in manufacturing, such as the alignment of the plate within the wing or the shrinking of the skin foil.

When the hinge is released, wings A, B, and C present different slopes but do not follow a trend that could be related to the differences in bending stiffness. In addition, the slopes presented by the tailored wings are almost the same and lower than the reference slope of wing B, contrary to the expected results seen in the locked condition. Finally, stall in the studied range is only found on wing $\mathrm{B}_{\mathrm{WI}}$, which is in agreement with this wing presenting the lowest stall angle in the locked condition and advancing stall in comparison to the reference wing B. However, since stall does not appear on the other wings in the studied range, it is not possible to study if all the trends observed in the locked condition are extended to the released condition.

\section{Dynamic Load: 1-Cosine Gusts}

The last part of the experiment studies the dynamic response of the wing when hit by 1-cosine gusts to assess the GLA performance of the FWT. Before addressing the results, the response of the system is characterized by studying the time delays introduced during the actuation process.

\section{System Characterization}

When studying the time response of the different wings, it is found that the release time of the hinge mechanism has a great impact on the FWT performance. For this reason, it has been considered necessary to characterize this response before addressing the GLA performance. There are two sources of possible time delay.

The first source of time delay originates from the Arduino requiring time to process the input and release the hinge. As seen in the diagram from Figure 14, the microprocessor adds a delay to release the hinge at the desired load. To know how precise this release is, the delay input is compared to the time between reading the trigger signal from the gust generator and reading the control signal obtained from the Arduino that indicates the position that the microcontroller is sending to the actuator. This delay is analyzed across all the test cases and amounts to an average delay of $2 \mathrm{~ms}$ with a standard deviation of $1.3 \mathrm{~ms}$.

The second source of the time delay originates from the time it takes between sending the signal to move the actuator and the structure to start moving. To study this delay, the release time is compared to the potentiometer position. The structure is considered to start reacting when the potentiometer position changes by more than $1^{\circ}$. A representation of this time delay is presented in Figure 18, which shows that the potentiometer does not start moving immediately after sending the signal to the actuator. It is found that this delay is $50 \mathrm{~ms}$ on average with a standard deviation of $7 \mathrm{~ms}$.

The importance of these delays depends on the gust frequency as quantified in Table 1 ll by converting the time delays into relative delays with respect to the gust period. The $2 \mathrm{~ms}$ delay introduced by the Arduino can be neglected for all gust cases, as it represents less than a $2 \%$ of the gust period. However, the $50 \mathrm{~ms}$ delay required for the structure to react becomes significant for the fast gusts. On the one hand, for the long gusts, $0.5 \mathrm{~Hz}$, the delay remains below

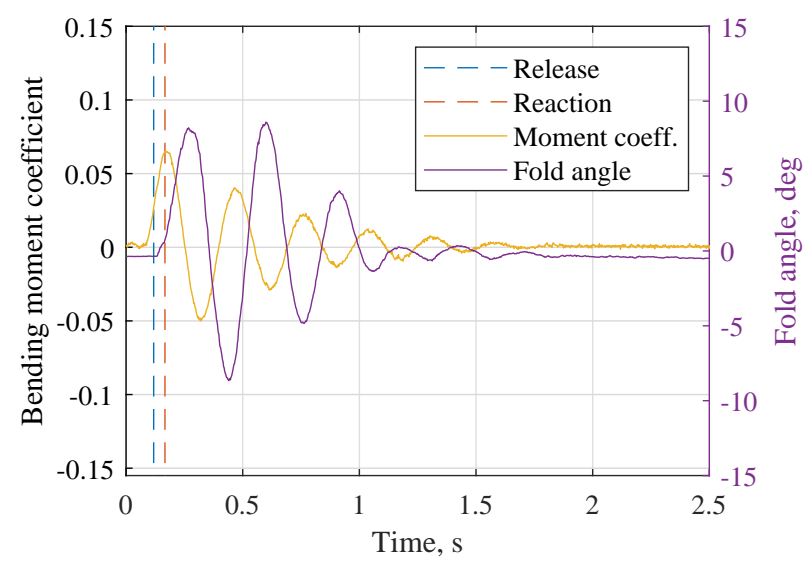

Fig. 18 Example of delay between actuator signal release and potentiometer response. Example of Wing A, $\alpha=0^{\circ}, 5 \mathrm{~Hz}$ gust and $50 \%$ release 
$5 \%$ of the gust period. On the other hand, for the shorter gusts, this delay represents more than $20 \%$ of the gust period for the $5 \mathrm{~Hz}$ and $8 \mathrm{~Hz}$ gusts. This can be seen in Figure 19, where an example of time response at $0.5 \mathrm{~Hz}$ is compared to an example at $8 \mathrm{~Hz}$. In the $0.5 \mathrm{~Hz}$ gust, when the hinge is released at $50 \%$ of the peak load, a $50 \mathrm{~ms}$ delay results in an effective release threshold of $60 \%$, while the same delay at $8 \mathrm{~Hz}$ leads to an effective release threshold of $90 \%$. Therefore, it is necessary to consider these delays when assessing the load alleviation performance of the wings.

Table 11 Comparison between gust periods and time delays introduced during the release

\begin{tabular}{cccccc}
\hline \hline \multicolumn{2}{c}{ Gust } & \multicolumn{2}{c}{2 ms delay } & \multicolumn{2}{c}{50 ms delay } \\
Frequency $[\mathrm{Hz}]$ & Period [ms] & Relative delay $[\%]$ & Phase delay $\left[{ }^{\circ}\right]$ & Relative delay $[\%]$ & Phase delay $\left[{ }^{\circ}\right]$ \\
\hline 0.5 & 2000 & 0.1 & 0.4 & 2.5 & 9 \\
1.5 & 667 & 0.3 & 1.1 & 7.5 & 27 \\
3.0 & 333 & 0.6 & 2.2 & 15 & 54 \\
3.5 & 286 & 0.7 & 2.5 & 17.5 & 63 \\
5.0 & 200 & 1.0 & 3.6 & 25 & 90 \\
8.0 & 125 & 1.6 & 5.8 & 40 & 144 \\
\hline \hline
\end{tabular}

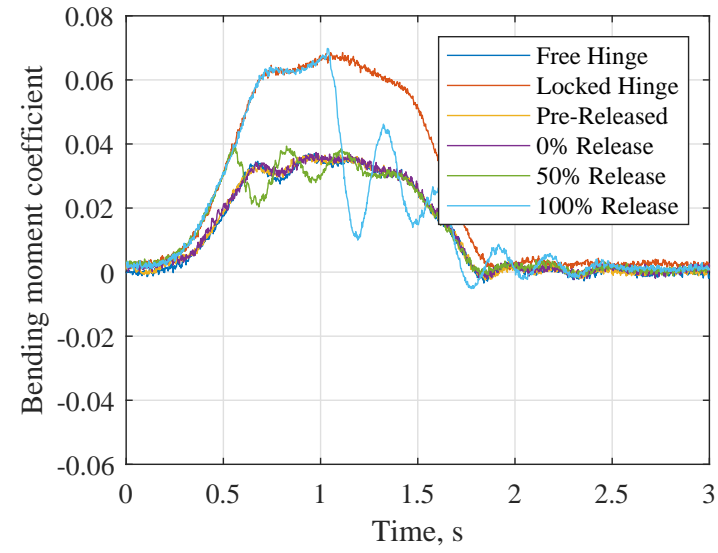

(a) Bending moment time response for $0.5 \mathrm{~Hz}$ gust

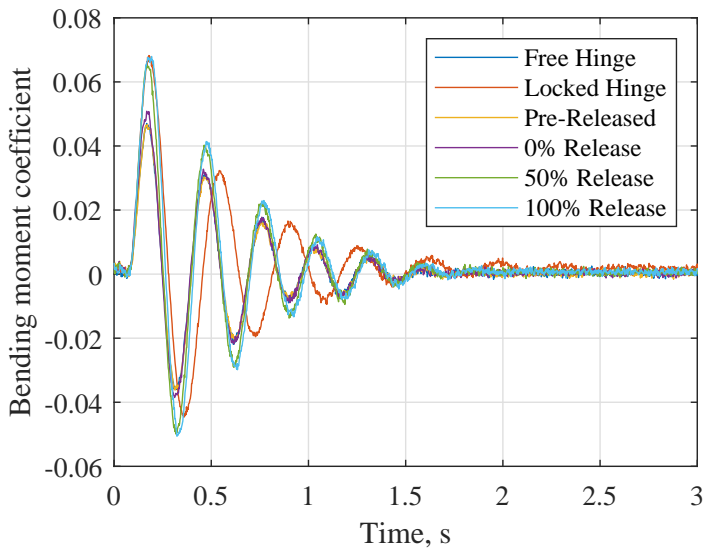

(b) Bending moment time response for $8 \mathrm{~Hz}$ gust

Fig. 19 Comparison of time response for different release thresholds at different gust frequencies. Example from Wing A at $\alpha=0^{\circ}$

\section{GLA Performance}

The GLA performance of the FWT is assessed by comparing the peak loads and RMS with the performance of the wing with a locked hinge. First, the relative peak loads at $\alpha=0^{\circ}$ are addressed to study the case in which no static load is applied. Then, the static load is included in the study of the peak loads at $\alpha=5^{\circ}$. Afterward, the introduction of oscillations is studied with the relative RMS. Similar to the peak loads investigation, first the case in which $\alpha=0^{\circ}$ is addressed, and then the study is concluded with the $\alpha=5^{\circ}$ case.

Figure 20 presents a comparison of the relative peak load alleviation between wings for different gust frequencies and release thresholds at $\alpha=0^{\circ}$. From these results, interesting remarks can be made regarding the release threshold and the peak loads when the hinge is released close to the peak load. First, the free hinge can produce a peak load reduction between 30\% and 50\%, depending on the gust frequency and the wing stiffness. In agreement with the expectations, the peak load reduction is reduced with the delay in the release, as the wing is allowed to achieve higher loads before the hinge is released. Nevertheless, when the hinge is released at $100 \%$ of the peak load, the FWT can increase the peak loads with respect to the locked-hinge condition. Notice also that this load increase depends on the gust frequency. For the $0.5 \mathrm{~Hz}$ gust, the peak increase can only be seen in the $100 \%$ release while, for the gust at $8 \mathrm{~Hz}$, the load enhancement is also observed at $50 \%$ release. Furthermore, it can be seen how the load alleviation 
for the $50 \%$ release is progressively reduced with the increase of the gust frequency: for $0.5 \mathrm{~Hz}$ gust, the alleviation is similar to the free-hinge condition while load enhancement can be observed for $8 \mathrm{~Hz}$ gusts. This change can be explained by the delays introduced by the release system. As seen before, the release threshold is higher than desired as a consequence of the delays and, depending on the gust frequency, the increase in the load threshold becomes more important. Therefore, the reduction in load alleviation seen with the increasing gust frequency is not directly caused by the gust frequency but by the change in release threshold.

When comparing the different wings, the wing B shows consistently lower load alleviation not only compared to the different stiffness wings, $\mathrm{A}$ and $\mathrm{C}$, but also to the tailored wings, $\mathrm{B}_{\mathrm{WI}}$ and $\mathrm{B}_{\mathrm{WO}}$. In addition, there is no significant difference between the tailored wings. Furthermore, exciting the wings at their natural frequencies does not seem to have an impact on the alleviation performance of the FWT. Even for wings B and C, which had the closest 1st bending natural frequencies to the ones used in the test, the results do not stand out. Overall, the load alleviation capabilities are similar between wings for all gust frequencies.

Next, the case with the static aerodynamic loads is considered by increasing the angle of attack to $\alpha=5^{\circ}$. Figure 21] presents the respective relative peak load comparison. Similar to the case without the static aerodynamic loads, the increase in peak loads still occurs for short gusts when the FWT is released beyond the 50\% load threshold. However, there is an important difference when considering the GLA improvement when the hinge is released before and at the instant when the gust hits the wing. The free-hinge condition allows a load alleviation similar to the $\alpha=0^{\circ}$ case. However, when the hinge is released in advance or at the $0 \%$ load threshold, the peak load can be reduced by up to 90\%. To understand these results, Figure 22a shows a comparison of the load response with a pre-released hinge and the respective response in locked-hinge and free-hinge conditions.

As seen in subsubsection III.B.3, there is a difference between the steady-state before the gust in locked-hinge condition and after the gust hits, when the hinge has already been released. When the hinge is released before the gust, the FWT starts moving towards the second steady-state, reducing the load. Once the gust hits, the increase in load caused by the gust is compensated by the load relief caused by the movement that the FWT starts when the hinge is released. As a result, the load barely goes above the initial steady load, leading to an almost $100 \%$ alleviation of the peak load.

To conclude the peak load assessment, the results at $\alpha=5^{\circ}$ are similar to those at $\alpha=0^{\circ}$ when looking at the effects of the different wing designs. This time, all the wings present similar performance. Even though the peak reductions seen at $\alpha=5^{\circ}$ are very promising, they are conditioned to a correct timing when releasing the hinge, since releasing at high load thresholds could aggravate the peak loads instead of alleviating them. In addition, there is an important drawback that cannot be seen from the relative peak loads. Figure 22b shows a gust response example in which, together with a slight decrease in peak load, there is an important increase in the magnitude and persistence of the oscillation of the signal when the hinge is released. The RMS of the signals is used to study the impact of the release on the oscillations.

Figure 23 shows the relative RMS with respect to the locked-hinge condition at $\alpha=0^{\circ}$. Contrary to what has been seen for the peak load, not only the hinge release affects the RMS but also the wing stiffness and tailoring are important. On the one hand, the RMS behavior with respect to the hinge release is similar to that seen on the peak loads. When the hinge is free, the RMS reduction can vary between $20 \%$ and $55 \%$ depending on the wing and gust frequency. The RMS reduction decrease with the increasing release threshold and, similarly to the peak loads, the oscillations can be aggravated with respect to the locked condition when the hinge is released at $100 \%$ of the peak load. For short gusts, this can also happen at 50\% release. On the other hand, it is also possible to see differences between the wings for gusts at $1.5 \mathrm{~Hz}, 5 \mathrm{~Hz}$, and $8 \mathrm{~Hz}$. Wing A presents lower RMS reduction than wings $\mathrm{B}$ and $\mathrm{C}$, while wing B presents the highest RMS reduction of all the wings. Both tailored wings, $\mathrm{B}_{\mathrm{WI}}$ and $\mathrm{B}_{\mathrm{WO}}$, present lower RMS reduction than wing $\mathrm{B}$, but wing $\mathrm{B}_{\mathrm{Wo}}$ achieves higher RMS reductions than $\mathrm{B}_{\mathrm{WI}}$. However, these trends cannot be observed for gust frequency of $0.5 \mathrm{~Hz}$ and the 1st bending natural frequency. For these cases, the RMS reduction is similar between wings.

When the static aerodynamic load is included in the case of $\alpha=5^{\circ}$, three main considerations remarks can be made from the results shown in Figure 24. First, the trends observed at $\alpha=0^{\circ}$ for the different wings in the $1.5 \mathrm{~Hz}, 5 \mathrm{~Hz}$, and $8 \mathrm{~Hz}$ gusts can still be observed in this case and, this time, they also appear for the $0.5 \mathrm{~Hz}$ gust and the 1 st bending natural frequency. Second, in contrast to the $\alpha=0^{\circ}$ case, the excitation at the natural frequency of the wings has an impact on the RMS at $\alpha=5^{\circ}$. When the wings are excited at their natural frequencies, the RMS reduction when the hinge is released in advance is lower than when it is released at the $0 \%$ threshold. Last, the increase in RMS seen for hinge releases at 50\% and $100 \%$ thresholds is amplified at $\alpha=5^{\circ}$ and can also be seen for the $5 \mathrm{~Hz}$ and $8 \mathrm{~Hz}$ gusts when released at $0 \%$ of the peak load. For these cases, the RMS can increase with respect to the locked hinge condition 
by up to $150 \%$, as in the example from Figure $22 b$.
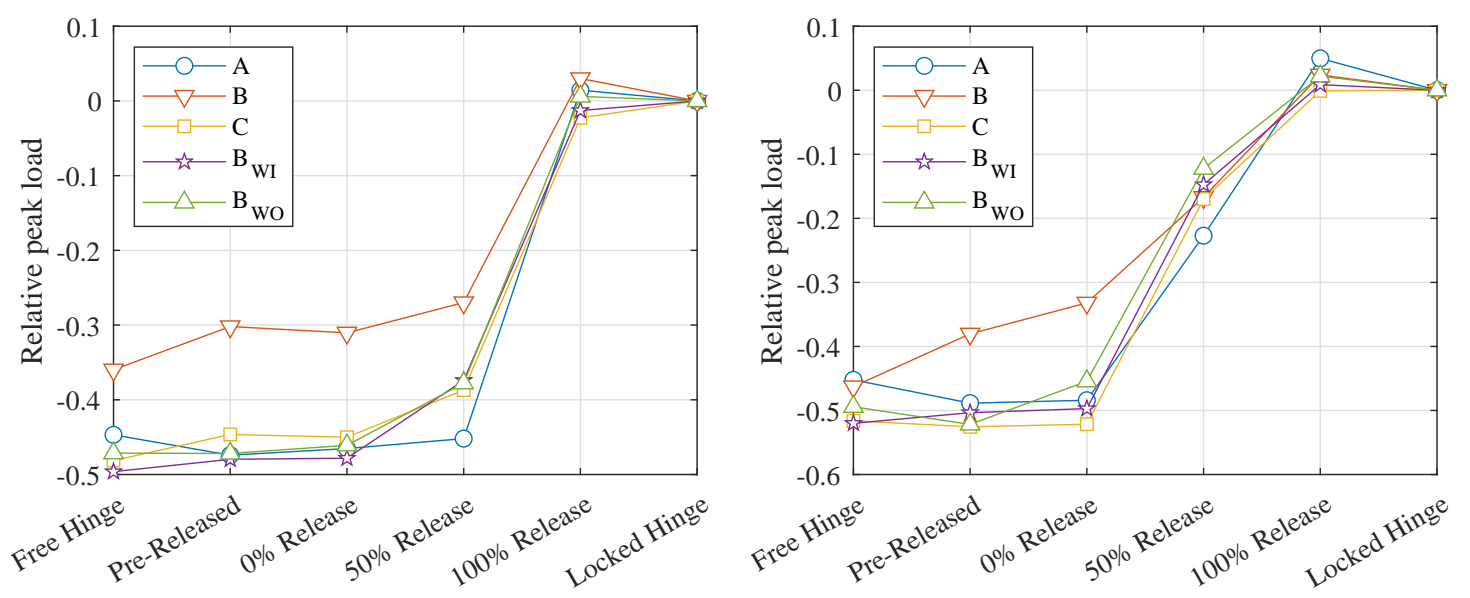

(a) $0.5 \mathrm{~Hz}$ Gust frequency

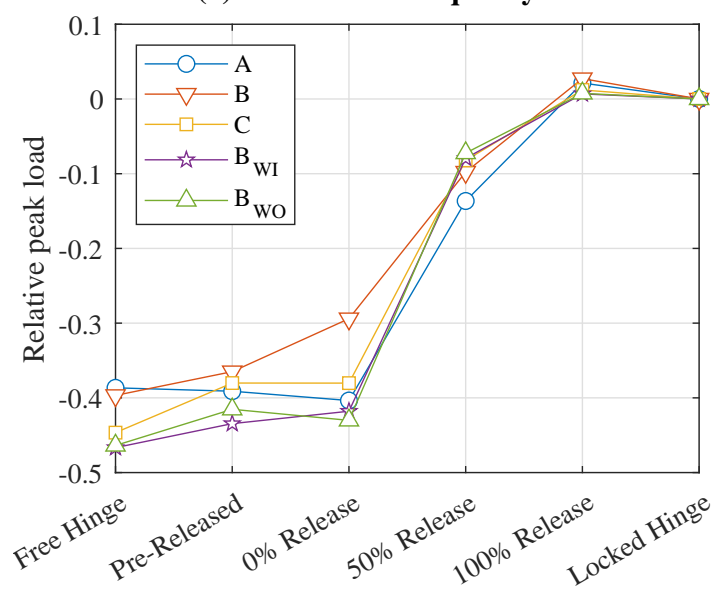

(b) 1st flapping natural frequency, $1.5 \mathrm{~Hz}$

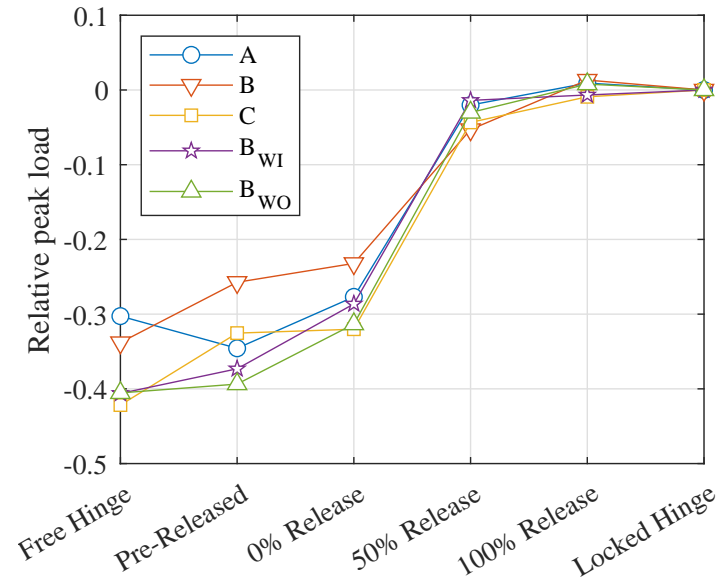

(c) 1st Bending natural frequency, $3.0 / 3.5 \mathrm{~Hz}$

(d) $5.0 \mathrm{~Hz}$ Gust frequency

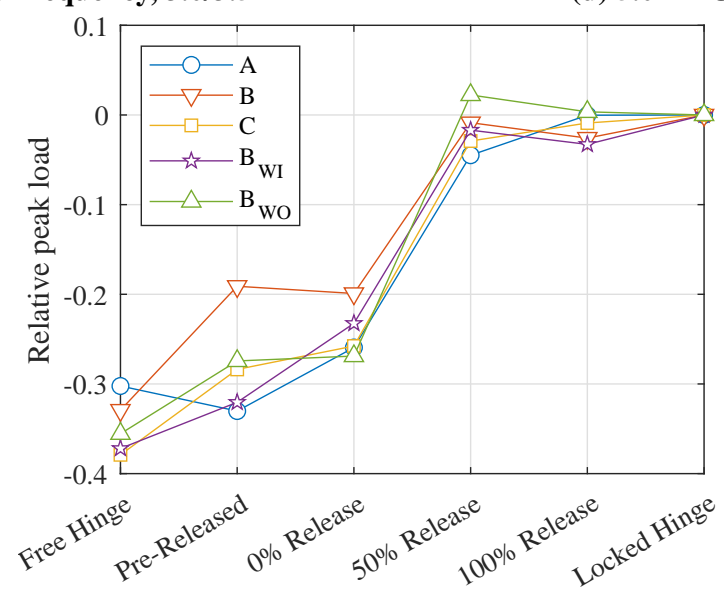

(e) $8.0 \mathrm{~Hz}$ Gust frequency

Fig. 20 Relative peak load reduction with respect to peak load in locked hinge condition for $\alpha=0^{\circ}$. 


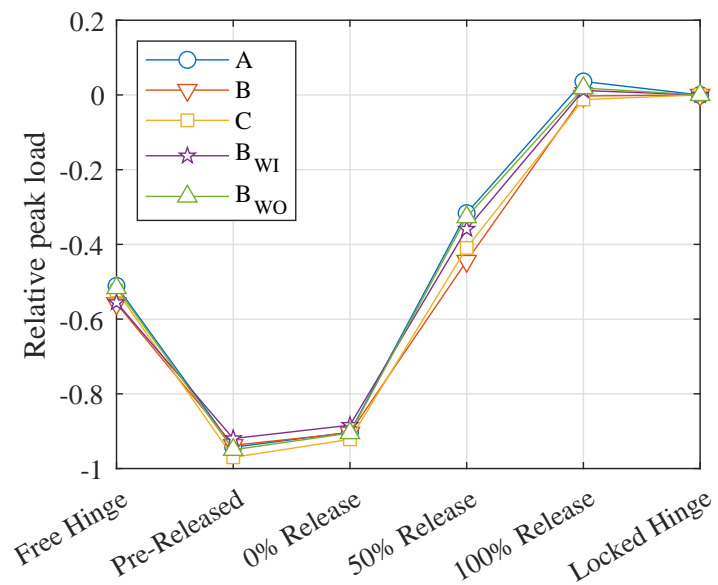

(a) $0.5 \mathrm{~Hz}$ Gust frequency

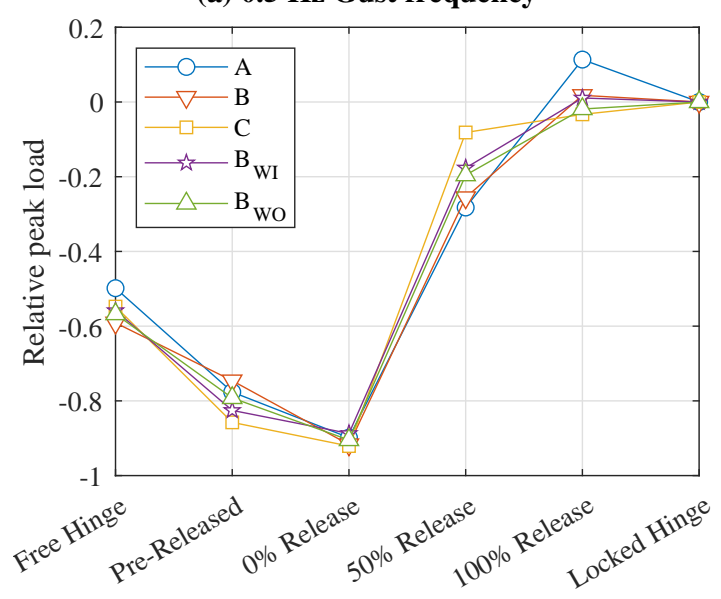

(c) 1st flapping natural frequency, 3.0/3.5 Hz

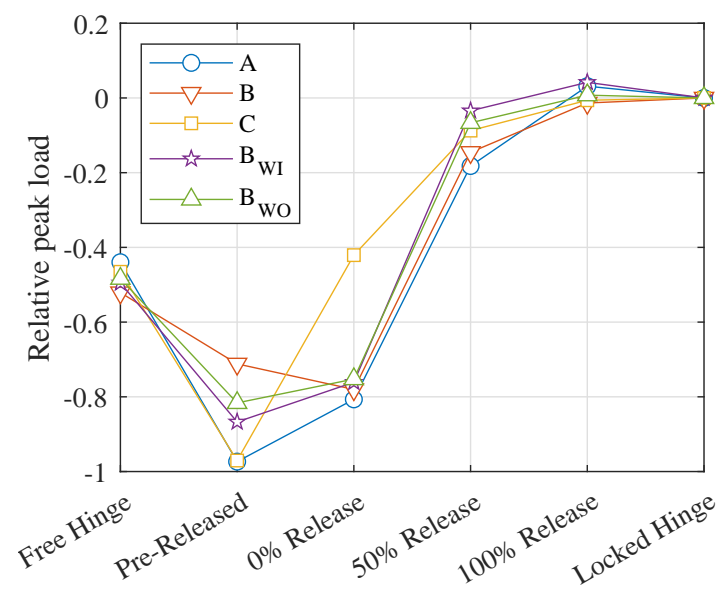

(b) 1st Bending natural frequency, $1.5 \mathrm{~Hz}$

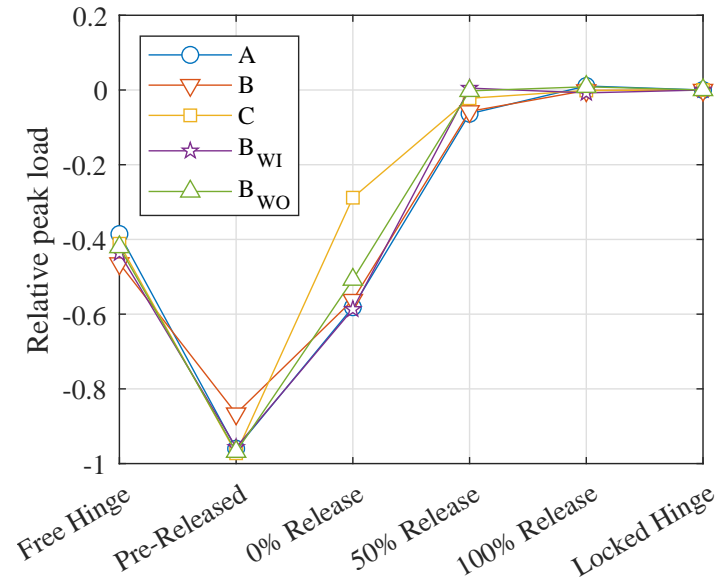

(d) $5.0 \mathrm{~Hz}$ Gust frequency

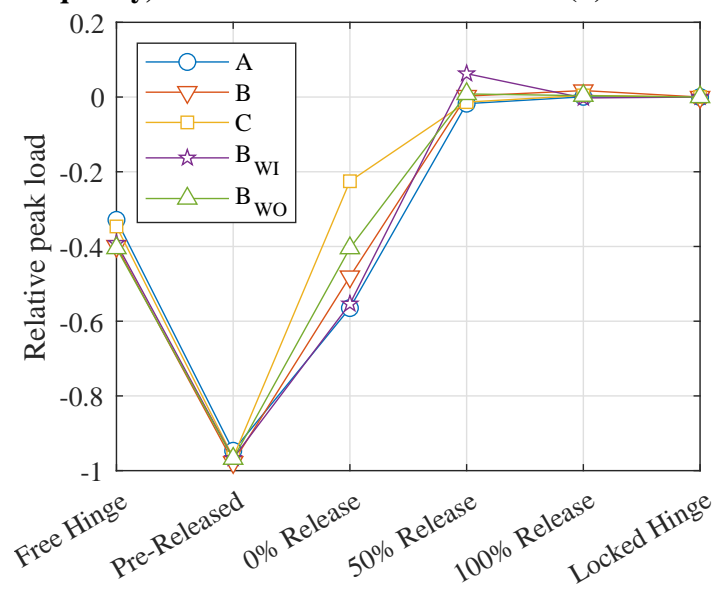

(e) $8.0 \mathrm{~Hz}$ Gust frequency

Fig. 21 Relative peak load reduction with respect to peak load in locked hinge condition for $\alpha=5^{\circ}$. 
To conclude the discussion of the results, it has been seen that it is important to release the hinge instantly after the gust is detected. When the hinge is released close to the peak load, the load can not only be aggravated but also introduce significant oscillations, which tend to persist for a considerable amount of time after the gust has passed the wing. The introduction of these oscillations can be critical, as they can be considered detrimental for reasons such as fatigue of the wing structure. However, the differences seen between the different wings show that it might be possible to minimize this impact with a proper structural design. In addition, other systems, like spoilers or the wingtip tab presented by Cheung et al. [6], could be combined to try to reduce these oscillations and further improve the GLA performance.
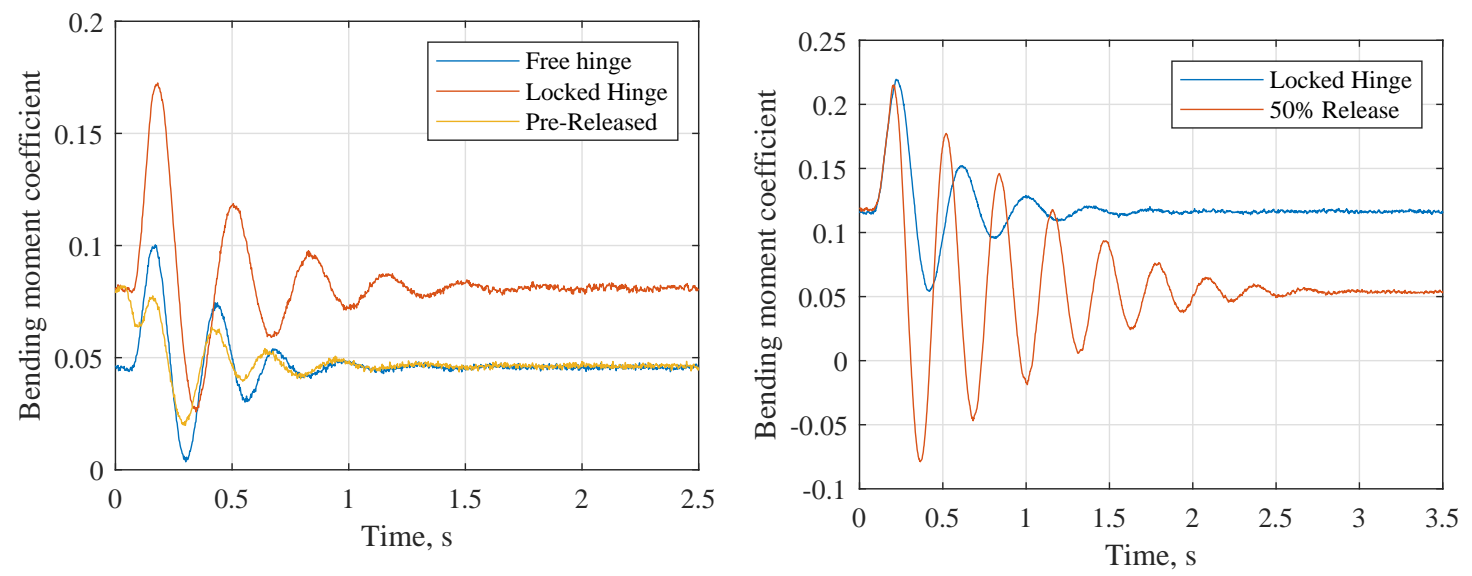

(a) Comparison of pre-release threshold with lockedhinge and free-hinge conditions at $\alpha=5^{\circ}$. Example from Wing $B$ for $8 \mathrm{~Hz}$ gust

(b) Comparison of $50 \%$ threshold with locked condition at $\alpha=5^{\circ}$. Example from Wing A for $5 \mathrm{~Hz}$ gust.

Fig. 22 Bending moment coefficient time response examples 


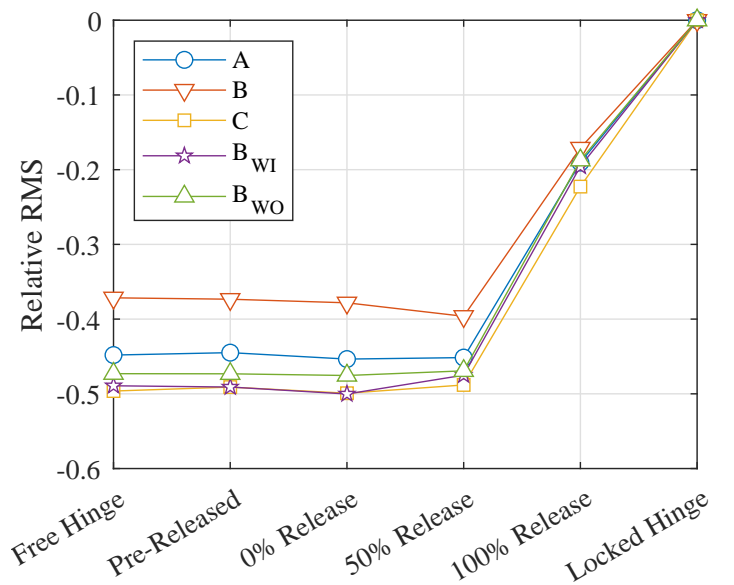

(a) $0.5 \mathrm{~Hz}$ Gust frequency

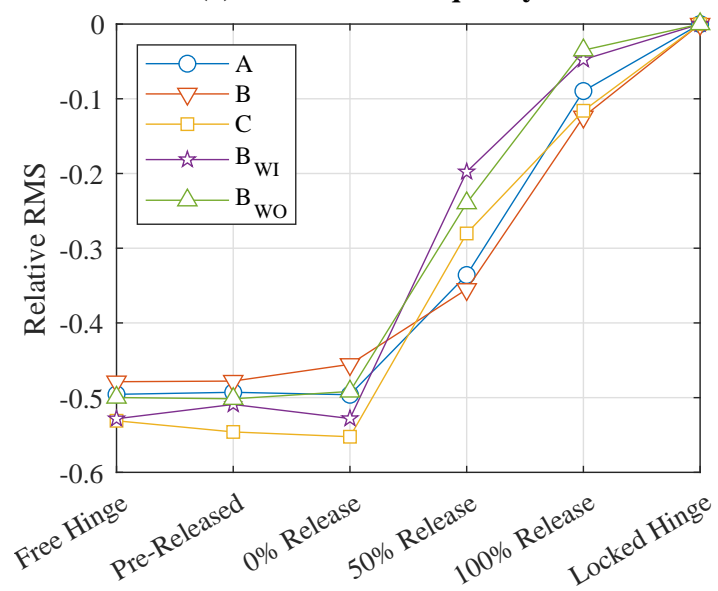

(c) 1st flapping natural frequency, 3.0/3.5 Hz

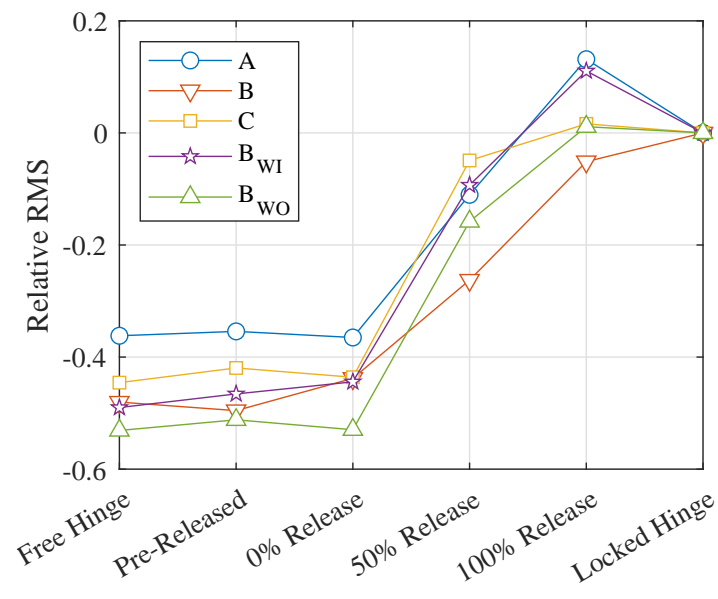

(b) 1st Bending natural frequency, $1.5 \mathrm{~Hz}$

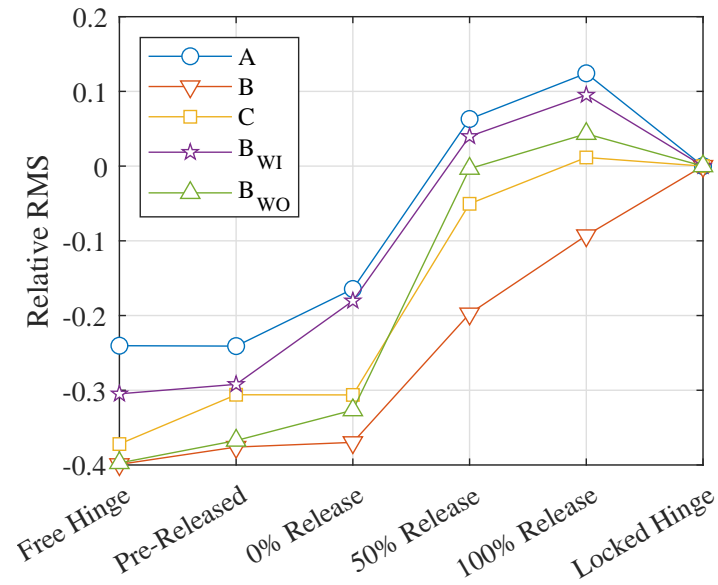

(d) $5.0 \mathrm{~Hz}$ Gust frequency

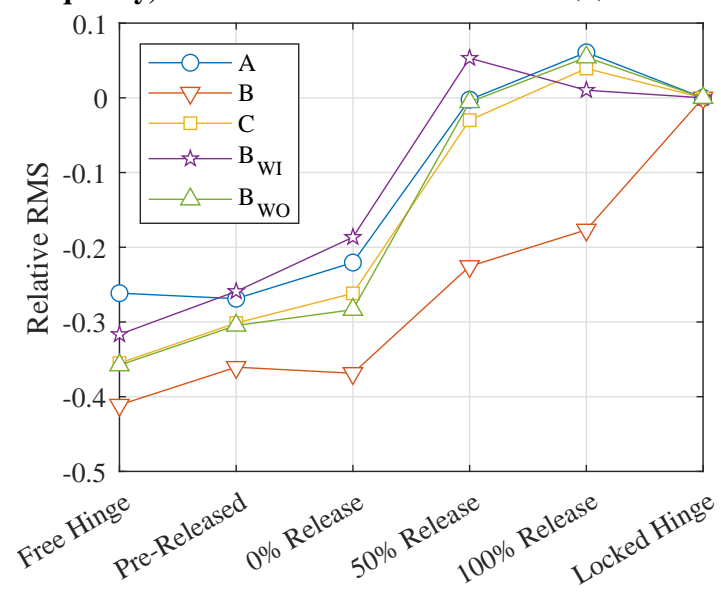

(e) $8.0 \mathrm{~Hz}$ Gust frequency

Fig. 23 Relative RMS reduction with respect to peak load in locked hinge condition for $\alpha=0^{\circ}$. 


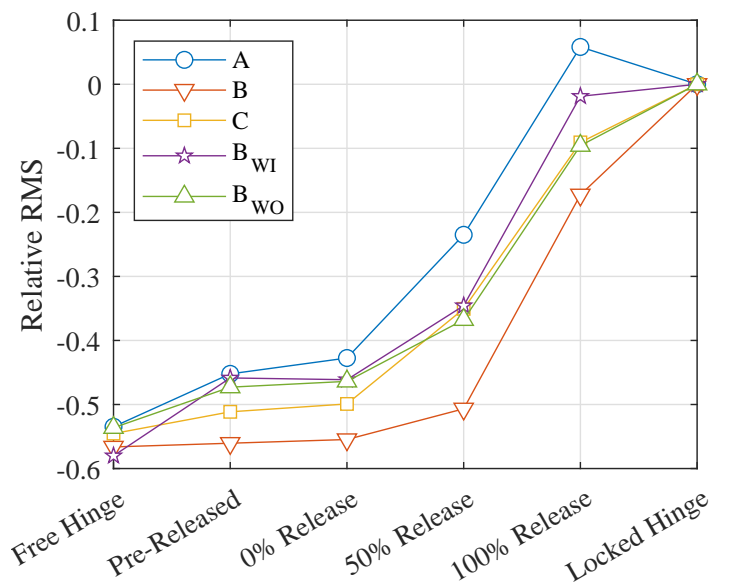

(a) $0.5 \mathrm{~Hz}$ Gust frequency

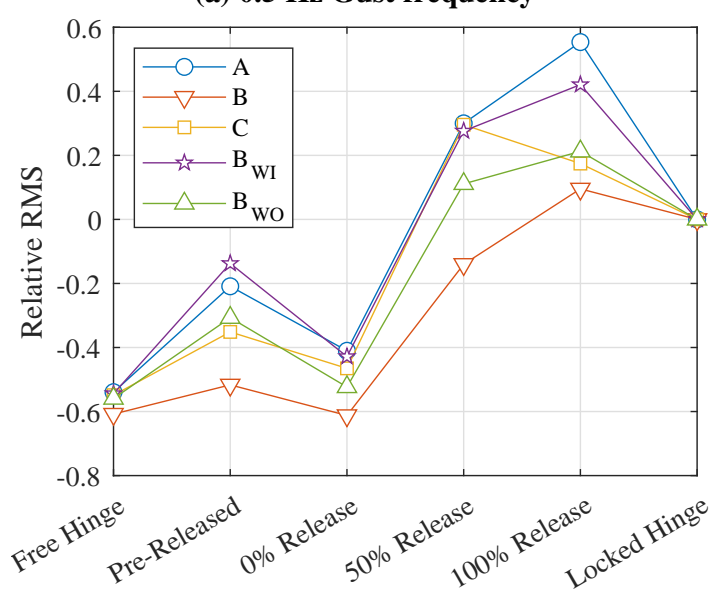

(c) 1st flapping natural frequency, 3.0/3.5 Hz

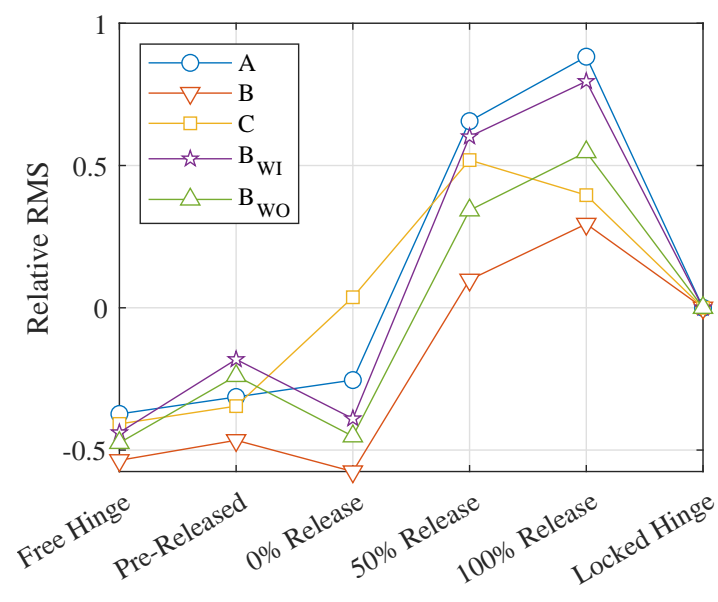

(b) 1st Bending natural frequency, $1.5 \mathrm{~Hz}$

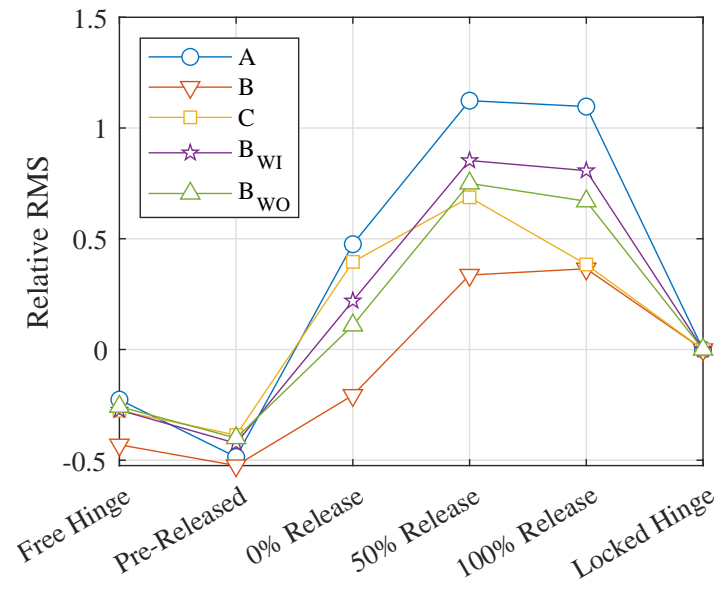

(d) $5.0 \mathrm{~Hz}$ Gust frequency

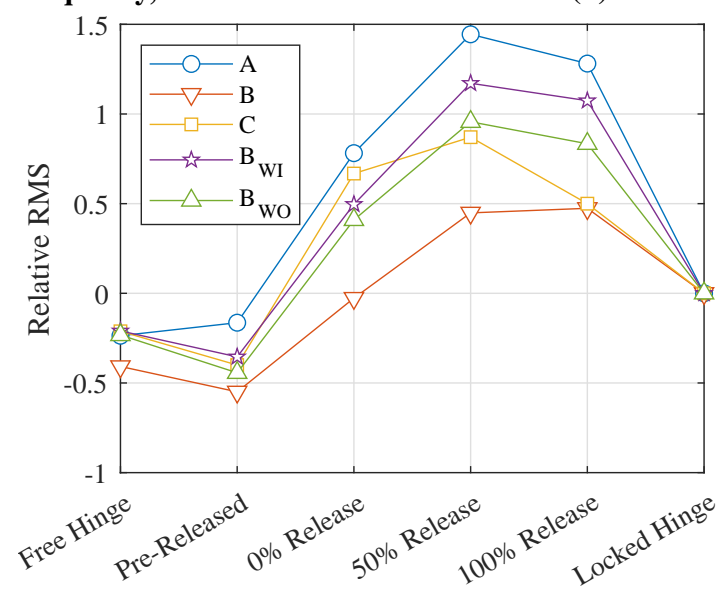

(e) $8.0 \mathrm{~Hz}$ Gust frequency

Fig. 24 Relative RMS reduction with respect to peak load in locked hinge condition for $\alpha=5^{\circ}$. 


\section{Conclusions}

This study has addressed the design and wind tunnel investigation of a wing model with a folding wingtip, equipped with a hinge mechanism to lock or release this articulation when desired. The wind tunnel experiment was used to assess the effects of the bending stiffness and tailoring of the main wing as well as the impact of the hinge release instant on the final gust load alleviation of the folding wingtip.

It was found that the linear model used as a sizing tool overpredicts the flutter speed by more than $40 \%$. In addition, when studying the effect of the hinge condition on flutter speed, a change in the flutter mechanism is found. This change would explain the unexpected effect of the tailoring on the flutter speed. While wash-in tailoring would delay flutter in conventional wings, it promotes it when the folding wingtip is free.

Regarding the loads, the $C_{B}$ polars show that the bending stiffness does not influence the static loads, leading to constant $C_{B, \alpha}$ across the stiffness range. However, tailoring does affect the loads. The wash-out tailoring decreases $C_{B, \alpha}$ due to the bending-twist coupling, which reduces the angle of attack when bending the wing. For the wash-in, this behavior is reversed.

Finally, there is no clear relationship between the peak load alleviation and the structural properties of the wing. Although in some test cases there is a clear difference in load alleviation, it is not consistent across the design space. On the other hand, there is a remarkable effect of the hinge release timing on the load alleviation. For statically unloaded conditions, corresponding to $\alpha=0^{\circ}$, releasing the hinge before the gust hits the wing is as effective as a released hinge. However, the later it is released the worse the load alleviation becomes, even increasing the peak loads when it is released close to the peak load. When there is a static load, in the case of $\alpha=5^{\circ}$, there is a significant difference wheter the hinge is released on time or not. When the hinge is released in advance, the difference between the static load in the locked-hinge condition and free-hinge condition makes the wingtip start moving before the gust hits, compensating the gust load and reducing the peak load by more than $90 \%$.

Although the dynamic release can alleviate the peak loads, it increases the oscillations of the wing, which can be considered detrimental for reasons such as fatigue of the wing structure. For $\alpha=0^{\circ}$ and releases close to the maximum load, these oscillations can become higher in amplitude and more persistent than if the hinge remained locked. For $\alpha=5^{\circ}$, this effect is magnified, leading to increments in the RMS values of more than $100 \%$ with respect to the locked condition.

These results emphasize the importance of detecting the gusts and being able to react on time. If the aircraft can detect them in advance and release the hinge before they hit the wing, the tradeoff between peak load reduction and oscillations can be positive. However, if the gust can only be detected when it hits the wing, the release and reaction time of the system needs to be small enough to avoid entering the "late release" region for which the loads can be worsened and the oscillations doubled.

Future work will be directed towards solving the modeling uncertainties seen during the experiment. On the one hand, ground vibration tests of the wing are planned to correlate the model with the actual wings and update the structural properties of the simulation to understand to what extent does the difference between the numerical model and experimental model affects the predictions. On the other hand, alternative solvers need to be investigated to account for the nonlinearities. For the gust response simulations, the results were limited to small wingtip deflections, hence a solver capable of representing the large deflections of the wingtip would improve the predictions. For the flutter analysis, the drop in critical speed and the limit cycle oscillations seen in the experiment show the complexity of the dynamic behavior of the system. Thus, a nonlinear solver could improve the flutter predictions.

To conclude, the present results open a few lines of new research. First, the already mentioned complexity of the system presents the necessity to understand the dynamic behavior of the folding wingtip and how it interacts with the main wing. The dynamic response of the wing in the free-hinge condition can lead to surprising results both in terms of aeroelastic stability margins as well as the aeroelastic response and gust load alleviation performance. Furthermore, the unexpected behavior of the aeroelastic tailoring when combined with the folding wingtip shows that the design rules applied for conventional wings might not be applicable for this concept and require further investigation. Finally, focusing on the increased persistence of the oscillations, it would be interesting to investigate the combination of the folding wingtip with other gust load alleviation control systems, such as ailerons or spoilers, to reduce the oscillations and further reduce the peak loads. 


\section{References}

[1] Anderson, J. D., Fundamentals of aerodynamics, $6^{\text {th }}$ ed., McGraw Hill, New York, 2017.

[2] Smith, M. H., Renzelmann, M. E., and Marx, A. D., "Folding Wing-Tip System," (U.S. Patent No. 5,381,986). U.S. Patent and Trademark Office, Jan. 17 1995. URL https://worldwide.espacenet.com/patent/search?q=pn\%3DUS5381986A.

[3] Wilson, T., Herring, M., Pattinson, J., Cooper, J., Castrichini, A., Ajaj, R., and Dhoru, H., "An Aircraft Wing With Moveable Wing Tip Device for Load Alleviation," (International Patent No. WO2017/118832Al). World Intellectual Property Organization, Jul. 13 2017. URL https://worldwide.espacenet.com/patent/search?q=pn\%3DW02017118832A1.

[4] Castrichini, A., Siddaramaiah, V. H., Calderon, D., Cooper, J., Wilson, T., and Lemmens, Y., "Preliminary Investigation of Use of Flexible Folding Wing Tips for Static and Dynamic Load Alleviation,” The Aeronautical Journal, Vol. 121, No. 1235, 2017, p. 73Ü94. https://doi.org/10.1017/aer.2016.108.

- [5] Cheung, R., Rezgui, D., Cooper, J., and Wilson, T., "Testing of a Hinged Wingtip Device for Gust Loads Alleviation,” Journal of Aircraft, Vol. 55, 2018, pp. 1-18. https://doi.org/10.2514/1.C034811.

[6] Cheung, R., Rezgui, D., Cooper, J., and Wilson, T., "Testing of Folding Wingtip for Gust Load Alleviation of Flexible High-Aspect-Ratio Wing," Journal of Aircraft, Vol. 57, No. 5, 2020, pp. 876-888. https://do1.org/10.2514/1.C035/32.

-[7] Castrichini, A., Hodigere Siddaramaiah, V., Calderon, D., Cooper, J., Wilson, T., and Lemmens, Y., "Nonlinear Folding Wing Tips for Gust Loads Alleviation,” Journal of Aircraft, Vol. 53, No. 5, 2016, pp. 1391-1399. https://doi.org/10.2514/1.C033474.

[8] Castrichini, A., Wilson, T., and Cooper, J., "On the Dynamic Release of the Semi Aeroelastic Wing-Tip Hinge Device," 6th RAeS Aircraft Structural Design Conference, 2018.

-[9] Dussart, G., Yusuf, S., and Lone, M., "Identification of In-Flight Wingtip Folding Effects on the Roll Characteristics of a Flexible Aircraft,” Aerospace, Vol. 6, 2019, p. 63. https://doi.org/10.3390/aerospace6060063.

[10] Healy, F., Cheung, R., Neofet, T., Lowenberg, M., Rezgui, D., Cooper, J., Castrichini, A., and Wilson, T., "Folding Wingtips for Improved Roll Performance," AIAA Scitech 2021 Forum, 2021. https://doi.org/10.2514/6.2021-1153.

[11] Wilson, T., Kirk, J., Hobday, J., and Castrichini, A., "Small Scale Flying Demonstration of Semi Aeroelastic Hinged Wing Tips," 19th International Forum on Aeroelasticity and Structural Dynamics (IFASD 2019), 2019.

[12] Avin, O., Raveh, D., Drachinsky, A., Ben-Shmuel, Y., and Tur, M., “An Experimental Benchmark of a Very Flexible Wing," AIAA Scitech 2021 Forum, 2021. https://doi.org/10.2514/6.2021-1709.

[13] Kassapoglou, C., Review of Classical Laminated Plate Theory, John Wiley \& Sons, Ltd, 2013, Chap. 3, pp. 33-53. https://doi.org/10.1002/9781118536933.ch3.

-[14] Dillinger, J. K. S., Klimmek, T., Abdalla, M. M., and Gürdal, Z., "Stiffness Optimization of Composite Wings with Aeroelastic Constraints," Journal of Aircraft, Vol. 50, No. 4, 2013, pp. 1159-1168. https://doi.org/10.2514/1.C032084.

-[15] Weisshaar, T., “Aeroelastic Tailoring - Creative Uses of Unusual Materials," 28th Structures, Structural Dynamics and Materials Conference, 1987. https://do1.org/10.2514/6.198/-9/6. 